\title{
Exotic triple-charm deuteronlike hexaquarks
}

\author{
Rui Chen, ${ }^{1,2,3}$ Fu-Lai Wang, ${ }^{1,2, *}$ Atsushi Hosaka, ${ }^{3, \dagger}$ and Xiang Liu ${ }^{1,2, *}$ \\ ${ }^{1}$ School of Physical Science and Technology, Lanzhou University, Lanzhou 730000, China \\ ${ }^{2}$ Research Center for Hadron and CSR Physics, Lanzhou University \\ and Institute of Modern Physics of CAS, Lanzhou 730000, China \\ ${ }^{3}$ Research Center for Nuclear Physics (RCNP), Osaka University, Ibaraki, Osaka 567-0047, Japan
}

(Received 22 April 2018; revised manuscript received 12 May 2018; published 8 June 2018)

\begin{abstract}
Adopting the one-boson-exchange model, we perform a systematic investigation of interactions between a doubly charmed baryon $\left(\Xi_{c c}\right)$ and an $S$-wave charmed baryon $\left(\Lambda_{c}, \Sigma_{c}^{(*)}\right.$, and $\left.\Xi_{c}^{(\prime, *)}\right)$. Both the $S-D$ mixing effect and coupled-channel effect are considered in this work. Our results suggest that there may exist several possible triple-charm deuteronlike hexaquarks. Meanwhile, we further study the interactions between a doubly charmed baryon and an $S$-wave anticharmed baryon. We find that a doubly charmed baryon and an $S$ wave anticharmed baryon can be easily bound together to form shallow molecular hexaquarks. These heavy flavor hexaquarks predicted here can be accessible at future experiment like LHCb.
\end{abstract}

DOI: 10.1103/PhysRevD.97.114011

\section{INTRODUCTION}

As a hot issue in hadron physics, exploring exotic hadronic matter is a research field full of challenges and opportunities, which is valuable to deepen our understanding of the nonperturbative behavior of quantum chromodynamics (QCD). The observations of $X Y Z$ charmoniumlike states, $P_{c}(4380)$ and $P_{c}(4450)$ [1] have stimulated abundant studies involved in hidden-charm tetraquarks and pentaquarks (see Refs. [2-4] for review) since 2003.

In general, there are two approaches to clearly identify exotic hadronic states: (1) we may conclude a hadronic state to be an exotic state if it has exotic spin-parity quantum number $J^{P C}$ like $0^{--}, 0^{+-}, 1^{-+}$and so on. A typical candidate is the observation of the $\pi_{1}(1400)$ from the COMPASS Collaboration [5] which has $J^{P C}=1^{-+}$, where obviously the $\pi_{1}(1400)$ cannot be grouped into conventional meson family. (2) If a hadronic state has a typical exotic quark configuration different from the conventional hadron, we may also definitely categorize it as an exotic state. The reported $X(5568)$ with fully openflavor content $s u \bar{c} \bar{d}[6,7]$ is a typical exotic candidate, although no significant is observed by the LHCb Collaboration [8], the CMS Collaboration of LHC [9], and the

\footnotetext{
*chenr15@1zu.edu.cn

hosaka@rcnp.osaka-u.ac.jp

*xiangliu@lzu.edu.cn
}

Published by the American Physical Society under the terms of the Creative Commons Attribution 4.0 International license. Further distribution of this work must maintain attribution to the author(s) and the published article's title, journal citation, and DOI. Funded by SCOAP ${ }^{3}$.
CDF Collaboration of Fermilab [10]. The above criteria provides us valuable hints to identify exotic hadronic states.

Very recently, a double-charm baryon $\Xi_{c c}^{++}(3621)$ was discovered by the LHCb Collaboration when analyzing the $\Lambda_{c}^{+} K^{-} \pi^{+} \pi^{-}$invariant mass spectrum [11]. The observed $\Xi_{c c}^{++}(3621)$ not only make the baryon family become complete, but also inspires our interest in exploring the interaction of it with other hadrons, which has a close relation to the exploration of exotic hadronic molecular states. After the observation of $\Xi_{c c}^{++}(3621)$, the interaction of two doubly charmed baryons was investigated in Ref. [12], and then the same authors predicted the possible hadronic molecules composed of the doubly charmed baryon and nucleon [13], which is involved in the interaction of a doubly charmed baryon and a nucleon. Recently, Chen, Hosaka, and Liu performed a study of triple-charm molecular pentaquarks by checking the interaction of a doubly charmed baryon and a charmed meson [14]. Along this line, it is natural to extend these former studies to the interaction of a doubly charmed baryon and a charmed baryon, which will be a main task of the present work.

As shown in Fig. 1, charmed baryons can be categorized as $\overline{3}_{F}$ and $6_{F}$ representations based on flavor symmetries of light quarks. $\overline{3}_{F}$ and $6_{F}$ correspond to the light quarks in flavor antisymmetry and symmetry, respectively. Spin-parity for an $S$-wave charmed baryon is either $1 / 2^{+}$or $3 / 2^{+}$. Here, we will discuss the $\Xi_{c c} \mathcal{B}\left(1 / 2^{+}\right)$interactions with $\mathcal{B}=\Lambda_{c} / \Sigma_{c} / \Xi_{c}^{(\prime)}$ and the $\Xi_{c c} \mathcal{B}^{*}\left(3 / 2^{+}\right)$interactions with $\mathcal{B}^{*}=\Sigma_{c}^{*} / \Xi_{c}^{*}$.

By examining the interaction of a doubly charmed baryon and a charmed baryon, we want to answer whether or not there exist possible triple-charm deuteronlike hexaquarks, which has the cccqqq configuration. Due to this typical configuration, these discussed triple-charm deuteronlike 


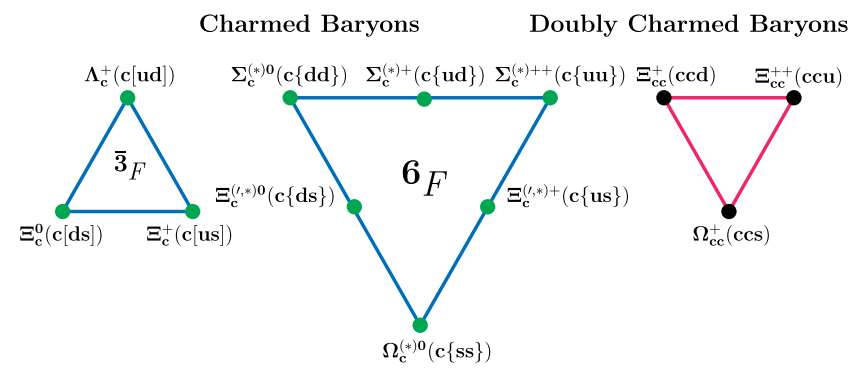

FIG. 1. Charmed baryons in $\overline{3}_{F}$ and $6_{F}$ representations and doubly charmed baryons. Here, we define $\left[q_{1} q_{2}\right]=\frac{1}{\sqrt{2}}\left(q_{1} q_{2}-\right.$ $\left.q_{2} q_{1}\right)$ and $\left\{q_{1} q_{2}\right\}=\frac{1}{\sqrt{2}}\left(q_{1} q_{2}+q_{2} q_{1}\right)$.

hexaquarks are typical exotic hadronic states apparently different from the conventional hadron. For achieving this goal, in this work we adopt the one-boson-exchange (OBE) model to get the effective potential of the interaction of a doubly charmed baryon and a charmed baryon, by which we may search for the corresponding bound state solutions. Finally, we will predict the existence of triple-charm deuteronlike hexaquarks. The present investigation provides crucial information to experimental search for possible triplecharm deuteronlike hexaquarks.

As an extension, exploring the interactions between a doubly charmed baryon and an $S$-wave anticharmed baryon will be carried out in this work. And then, we further predict possible shallow molecular hexaquarks composed of a doubly charmed baryon and an $S$-wave anticharmed baryon.

This paper is organized as follows. In Sec. II, the detailed calculation of effective potential related to the interaction between an $S$-wave doubly charmed baryon and an $S$-wave charmed baryon will be given, and the corresponding numerical results will be presented in Sec. III. Finally, we will give a short summary in Sec. IV.

\section{INTERACTIONS}

According to the heavy quark symmetry, chiral symmetry and hidden local symmetry [15,16], effective Lagrangians for charmed baryons and light meson interactions are constructed as

$$
\begin{gathered}
\mathcal{L}_{\mathcal{B}_{\overline{3}}}=l_{B}\left\langle\overline{\mathcal{B}}_{\overline{3}} \sigma \mathcal{B}_{\overline{3}}\right\rangle+i \beta_{B}\left\langle\overline{\mathcal{B}}_{\overline{3}} v^{\mu}\left(\mathcal{V}_{\mu}-\rho_{\mu}\right) \mathcal{B}_{\overline{3}}\right\rangle, \\
\mathcal{L}_{\mathcal{B}_{6}}=l_{S}\left\langle\overline{\mathcal{S}}_{\mu} \sigma \mathcal{S}^{\mu}\right\rangle-\frac{3}{2} g_{1} \varepsilon^{\mu \nu \lambda \kappa} v_{\kappa}\left\langle\overline{\mathcal{S}}_{\mu} \mathcal{A}_{\nu} \mathcal{S}_{\lambda}\right\rangle \\
+i \beta_{S}\left\langle\overline{\mathcal{S}}_{\mu} v_{\alpha}\left(\mathcal{V}^{\alpha}-\rho^{\alpha}\right) \mathcal{S}^{\mu}\right\rangle+\lambda_{S}\left\langle\overline{\mathcal{S}}_{\mu} F^{\mu \nu}(\rho) \mathcal{S}_{\nu}\right\rangle \\
\mathcal{L}_{\mathcal{B}_{\overline{3}} \mathcal{B}_{6}}=i g_{4}\left\langle\overline{\mathcal{S}}^{\mu} \mathcal{A}_{\mu} \mathcal{B}_{\overline{3}}\right\rangle+i \lambda_{I} \varepsilon^{\mu \nu \lambda \kappa} v_{\mu}\left\langle\overline{\mathcal{S}}_{\nu} F_{\lambda \kappa} \mathcal{B}_{\overline{3}}\right\rangle+\text { H.c. }
\end{gathered}
$$

Here, $\mathcal{A}_{\mu}$ and $\mathcal{V}_{\mu}$ stand for the axial current and vector current, respectively

$$
\begin{aligned}
& \mathcal{A}_{\mu}=\frac{1}{2}\left(\xi^{\dagger} \partial_{\mu} \xi-\xi \partial_{\mu} \xi^{\dagger}\right)=\frac{i}{f_{\pi}} \partial_{\mu} P+\cdots, \\
& \mathcal{V}_{\mu}=\frac{1}{2}\left(\xi^{\dagger} \partial_{\mu} \xi+\xi \partial_{\mu} \xi^{\dagger}\right)=\frac{i}{2 f_{\pi}^{2}}\left[P, \partial_{\mu} P\right]+\cdots
\end{aligned}
$$

with $\xi=\exp \left(i P / f_{\pi}\right) \cdot f_{\pi}$ is the pion decay constant with a value of $132 \mathrm{MeV}$. $\rho^{\mu}=i g_{V} V^{\mu} / \sqrt{2}, F^{\mu \nu}(\rho)=\partial^{\mu} \rho^{\nu}-$ $\partial^{\nu} \rho^{\mu}+\left[\rho^{\mu}, \rho^{\nu}\right]$. In Eqs. (2.2) and (2.3), we define a superfield $\mathcal{S}$, which is expressed as a combination of $\mathcal{B}_{6}$ with $J^{P}=1 / 2^{+}$and $\mathcal{B}_{6}^{*}$ with $J^{P}=3 / 2^{+}, \mathcal{S}_{\mu}=$ $-\sqrt{\frac{1}{3}}\left(\gamma_{\mu}+v_{\mu}\right) \gamma^{5} \mathcal{B}_{6}+\mathcal{B}_{6 \mu}^{*}$. Matrices for $\mathcal{B}_{\overline{3}}$ and $\mathcal{B}_{6}^{(*)}$ are

$$
\begin{aligned}
\mathcal{B}_{\overline{3}} & =\left(\begin{array}{ccc}
0 & \Lambda_{c}^{+} & \Xi_{c}^{+} \\
-\Lambda_{c}^{+} & 0 & \Xi_{c}^{0} \\
-\Xi_{c}^{+} & -\Xi_{c}^{0} & 0
\end{array}\right), \\
\mathcal{B}_{6}^{(*)} & =\left(\begin{array}{ccc}
\Sigma_{c}^{(*)++} & \frac{\Sigma_{c}^{(*)+}}{\sqrt{2}} & \frac{\Xi_{c}^{\left(\prime^{\prime} *\right)+}}{\sqrt{2}} \\
\frac{\Sigma_{c}^{(*)+}}{\sqrt{2}} & \Sigma_{c}^{(*) 0} & \frac{\Xi_{c}^{(, *) 0}}{\sqrt{2}} \\
\frac{\Xi_{c}^{(, * *)+}}{\sqrt{2}} & \frac{\Xi_{c}^{(, *) 0}}{\sqrt{2}} & \Omega_{c}^{(*) 0}
\end{array}\right) .
\end{aligned}
$$

In addition, matrices for pseudoscalar mesons $P$ and vector mesons $V$ are written as

$$
\begin{aligned}
& P=\left(\begin{array}{ccc}
\frac{\pi^{0}}{\sqrt{2}}+\frac{\eta}{\sqrt{6}} & \pi^{+} & K^{+} \\
\pi^{-} & -\frac{\pi^{0}}{\sqrt{2}}+\frac{\eta}{\sqrt{6}} & K^{0} \\
K^{-} & \bar{K}^{0} & -\sqrt{\frac{2}{3} \eta}
\end{array}\right), \\
& V=\left(\begin{array}{ccc}
\frac{\rho^{0}}{\sqrt{2}}+\frac{\omega^{\prime}}{\sqrt{6}} & \rho^{+} & K^{*+} \\
\rho^{-} & -\frac{\rho^{0}}{\sqrt{2}}+\frac{\omega^{\prime}}{\sqrt{6}} & K^{* 0} \\
K^{*-} & \bar{K}^{* 0} & -\sqrt{\frac{2}{3}} \omega^{\prime}
\end{array}\right) .
\end{aligned}
$$

By expanding Eqs. (2.1)-(2.3), the concrete effective Lagrangians are

$$
\begin{gathered}
\mathcal{L}_{\mathcal{B}_{\overline{3}} \mathcal{B}_{\overline{3}} \sigma}=l_{B}\left\langle\overline{\mathcal{B}}_{\overline{3}} \sigma \mathcal{B}_{\overline{3}}\right\rangle, \\
\mathcal{L}_{\mathcal{B}_{6}^{(*)} \mathcal{B}_{6}^{(*)} \sigma}=-l_{S}\left\langle\overline{\mathcal{B}}_{6} \sigma \mathcal{B}_{6}\right\rangle+l_{S}\left\langle\overline{\mathcal{B}}_{6 \mu}^{*} \sigma \mathcal{B}_{6}^{* \mu}\right\rangle, \\
\mathcal{L}_{\mathcal{B}_{\overline{3}} \mathcal{B}_{\overline{3}} V}=\frac{1}{\sqrt{2}} \beta_{B} g_{V}\left\langle\overline{\mathcal{B}}_{\overline{3}} v \cdot V \mathcal{B}_{\overline{3}}\right\rangle, \\
\mathcal{L}_{\mathcal{B}_{6}^{(*)} \mathcal{B}_{6}^{(*)} P}=i \frac{g_{1}}{2 f_{\pi}} \varepsilon^{\mu \nu \lambda \kappa} v_{\kappa}\left\langle\overline{\mathcal{B}}_{6} \gamma_{\mu} \gamma_{\lambda} \partial_{\nu} P \mathcal{B}_{6}\right\rangle \\
-i \frac{3 g_{1}}{2 f_{\pi}} \varepsilon^{\mu \nu \lambda \kappa} v_{\kappa}\left\langle\overline{\mathcal{B}}_{6 \mu}^{*} \partial_{\nu} P \mathcal{B}_{6 \lambda}^{*}\right\rangle \\
+i \frac{\sqrt{3}}{2} \frac{g_{1}}{f_{\pi}} v_{\kappa} \varepsilon^{\mu \nu \lambda \kappa}\left\langle\overline{\mathcal{B}}_{6 \mu}^{*} \partial_{\nu} P \gamma_{\lambda} \gamma^{5} \mathcal{B}_{6}\right\rangle+\text { H.c. },
\end{gathered}
$$




$$
\begin{gathered}
\mathcal{L}_{\mathcal{B}_{6}^{(*)} \mathcal{B}_{6}^{(*)} V=-} \frac{\beta_{S} g_{V}}{\sqrt{2}}\left\langle\overline{\mathcal{B}}_{6} v \cdot V \mathcal{B}_{6}\right\rangle-i \frac{\lambda g_{V}}{3 \sqrt{2}}\left\langle\overline{\mathcal{B}}_{6} \gamma_{\mu} \gamma_{\nu}\left(\partial^{\mu} V^{\nu}-\partial^{\nu} V^{\mu}\right) \mathcal{B}_{6}\right\rangle-\frac{\beta_{S} g_{V}}{\sqrt{6}}\left\langle\overline{\mathcal{B}}_{6 \mu}^{*} v \cdot V\left(\gamma^{\mu}+v^{\mu}\right) \gamma^{5} \mathcal{B}_{6}\right\rangle \\
-i \frac{\lambda_{S} g_{V}}{\sqrt{6}}\left\langle\overline{\mathcal{B}}_{6 \mu}^{*}\left(\partial^{\mu} V^{\nu}-\partial^{\nu} V^{\mu}\right)\left(\gamma_{\nu}+v_{\nu}\right) \gamma^{5} \mathcal{B}_{6}\right\rangle+\frac{\beta_{S} g_{V}}{\sqrt{2}}\left\langle\overline{\mathcal{B}}_{6 \mu}^{*} v \cdot V \mathcal{B}_{6}^{* \mu}\right\rangle+i \frac{\lambda_{S} g_{V}}{\sqrt{2}}\left\langle\overline{\mathcal{B}}_{6 \mu}^{*}\left(\partial^{\mu} V^{\nu}-\partial^{\nu} V^{\mu}\right) \mathcal{B}_{6 \nu}^{*}\right\rangle+\text { H.c. }, \\
\mathcal{L}_{\mathcal{B}_{\overline{3}} \mathcal{B}_{6}^{(*)} V}=-\frac{\lambda_{I} g_{V}}{\sqrt{6}} \varepsilon^{\mu \nu \lambda \kappa} v_{\mu}\left\langle\overline{\mathcal{B}}_{6} \gamma^{5} \gamma_{\nu}\left(\partial_{\lambda} V_{\kappa}-\partial_{\kappa} V_{\lambda}\right) \mathcal{B}_{\overline{3}}\right\rangle-\frac{\lambda_{I} g_{V}}{\sqrt{2}} \varepsilon^{\mu \nu \lambda \kappa} v_{\mu}\left\langle\overline{\mathcal{B}}_{6 \nu}^{*}\left(\partial_{\lambda} V_{\kappa}-\partial_{\kappa} V_{\lambda}\right) \mathcal{B}_{\overline{3}}\right\rangle+\text { H.c. } \\
\mathcal{L}_{\mathcal{B}_{\overline{3}} \mathcal{B}_{6}^{(*)} P}=-\sqrt{\frac{1}{3}} \frac{g_{4}}{f_{\pi}}\left\langle\overline{\mathcal{B}}_{6} \gamma^{5}\left(\gamma^{\mu}+v^{\mu}\right) \partial_{\mu} P \mathcal{B}_{\overline{3}}\right\rangle-\frac{g_{4}}{f_{\pi}}\left\langle\overline{\mathcal{B}}_{6 \mu}^{*} \partial^{\mu} P \mathcal{B}_{\overline{3}}\right\rangle+\text { H.c. }
\end{gathered}
$$

Suppose the interaction between heavy and light quarks is negligible, effective Lagrangians for the $S$-wave doubly charmed baryons and light meson interactions can be constructed as

$$
\begin{gathered}
\mathcal{L}_{\Xi_{c c} \Xi_{c c} \sigma}=g_{\sigma} \bar{\Xi}_{c c} \sigma \Xi_{c c}, \\
\mathcal{L}_{\Xi_{c c} \Xi_{c c} P}=g_{\pi} \bar{\Xi}_{c c} i \gamma_{5} P \Xi_{c c}, \\
\mathcal{L}_{\Xi_{c c} \Xi_{c c} V}=h_{v} \bar{\Xi}_{c c} \gamma_{\mu} V^{\mu} \Xi_{c c}+\frac{f_{v}}{2 M_{\Xi_{c c}}} \bar{\Xi}_{c c} \sigma_{\mu \nu} \partial^{\mu} V^{\nu} \Xi_{c c} .
\end{gathered}
$$

To obtain consistent coupling constants in these two kinds of effective Lagrangians, we would like to borrow the experience from the nucleon-nucleon interaction, which has the form of

$$
\begin{aligned}
\mathcal{L}_{N}= & g_{\sigma N N} \bar{N} \sigma N+\sqrt{2} g_{\pi N N} \bar{N} i \gamma_{5} P N \\
& +\sqrt{2} g_{\rho N N} \bar{N} \gamma_{\mu} V^{\mu} N+\frac{f_{\rho N N}}{\sqrt{2} m_{N}} \bar{N} \sigma_{\mu \nu} \partial^{\mu} V^{\nu} N .
\end{aligned}
$$

All of the coupling constants for the charmed baryon, doubly charmed baryon, and nucleon sectors are related in the quark level; the detailed derivations were given in Refs. [12,15]. In Table I, we finally summarize the values of all of the coupling constants and hadron masses adopted in the following calculations.

Under a Breit approximation, the effective potentials in momentum space is related to the corresponding scattering amplitude, i.e.,

$$
\mathcal{V}_{E}^{h_{1} h_{2} \rightarrow h_{3} h_{4}}(\boldsymbol{q})=-\frac{\mathcal{M}\left(h_{1} h_{2} \rightarrow h_{3} h_{4}\right)}{\sqrt{\prod_{i} 2 M_{i} \prod_{f} 2 M_{f}}} .
$$

$\mathcal{M}\left(h_{1} h_{2} \rightarrow h_{3} h_{4}\right), M_{i}$, and $M_{f}$ correspond to the scattering amplitude for the $h_{1} h_{2} \rightarrow h_{3} h_{4}$ process, the masses of the initial states $\left(h_{1}, h_{2}\right)$ and final states $\left(h_{3}, h_{4}\right)$, respectively. For the effective potentials in coordinate space $\mathcal{V}(r)$, it is obtained by performing a Fourier transformation,

$$
\mathcal{V}_{E}^{h_{1} h_{2} \rightarrow h_{3} h_{4}}(r)=\int \frac{d^{3} \boldsymbol{q}}{(2 \pi)^{3}} e^{i \boldsymbol{q} \cdot r} \mathcal{V}_{E}^{h_{1} h_{2} \rightarrow h_{3} h_{4}}(\boldsymbol{q}) \mathcal{F}^{2}\left(q^{2}, m_{E}^{2}\right) .
$$

Because the discussed hadrons are not pointlike particles, here, we introduce a monopole form factor ${ }^{1} \mathcal{F}\left(q^{2}, m_{E}^{2}\right)=$ $\left(\Lambda^{2}-m_{E}^{2}\right) /\left(\Lambda^{2}-q^{2}\right)$ at each interaction vertex, which is often adopted to study the nucleon-nucleon interaction $[21,22] . \Lambda, m_{E}$, and $q$ stand for cutoff, mass, and fourmomentum of exchanged mesons, respectively. In general, cutoff $\Lambda$ is related to the typical hadronic scale or the intrinsic size of hadrons. In our former paper [14], we reproduced the bound state property for the deuteron with the same parameters, by employing the cutoff $\Lambda=0.862 \mathrm{GeV}$. Assuming that the intrinsic size of hadrons are similar to that of the nucleon, we employ in the present study the cutoff $\Lambda$ of the same order around $1 \mathrm{GeV}$.

Here, we need to emphasize that all of the interaction strengths determined from the nucleon-nucleon interaction, and the only one phenomenological parameter, cutoff $\Lambda$, is estimated from the deuteron. In the following calculation, we will vary cutoff $\Lambda$ from 0.8 to $5.0 \mathrm{GeV}$ to search for the loosely bound solutions. The bound state with its cutoff close to $1 \mathrm{GeV}$ may be the possible molecular candidate.

To deduce total effective potentials, we need further construct wave functions for all of the discussed systems, which include the spin-orbit wave function, the flavor wave function, and the spatial wave function. In Table II, the flavor wave functions are collected.

Since the $S-D$ mixing effect is considered in this work, their spin-orbit wave functions include

\footnotetext{
${ }^{1}$ In general, the other kinds of form factor (like dipole form factor and exponential type form factor) are also adopted to discuss the hadron-hadron interaction. Once expanded, these form factors in the powers of $q^{2} / \Lambda^{2}, \mathcal{F}\left(q^{2}\right) \sim 1+c_{1} \times q^{2} / \Lambda^{2}+$ $c_{2} \times\left(q^{2} / \Lambda^{2}\right)^{2}+\cdots$, different form factors correspond to different sets of coefficients. Nevertheless, in the low-momentum limit, all of these different coefficients can be absorbed into the interactive strengths. Since the physics of molecular states is essentially lowmomentum physics, numerical results are quite similar by suitably choosing cutoffs and coupling constants.
} 
TABLE I. A summary of coupling constants and hadron masses adopted in our calculations. Here, the values relevant to the nucleonnucleon interactions are given in Refs. [17-19]. Masses of the hadrons involved in our study are taken from the Particle Data Group [20]. Unit of hadrons masses is $\mathrm{MeV}$.

\begin{tabular}{llll}
\hline \hline$\frac{g_{\sigma N N}^{2}}{4 \pi}=5.69$ & $\frac{g_{\pi N N}^{2}}{4 \pi}=13.60$ & $\frac{g_{\rho N N}^{2}}{4 \pi}=0.84$ & $\frac{f_{\rho N N}}{g_{\rho N N}}=6.10$ \\
$l_{S}=-2 l_{B}=-\frac{2}{3} g_{\sigma N N}$ & $g_{1}=\frac{2 \sqrt{2}}{3} g_{4}=-\frac{2 \sqrt{2} f_{\pi} g_{\pi N N}}{5 M_{N}}$ & $\beta_{S} g_{V}=-2 \beta_{B} g_{V}=-4 g_{\rho N N}$ & $\lambda_{S} g_{V}=-\sqrt{8} \lambda_{I} g_{V}=-\frac{6\left(g_{\rho N N}+f_{\rho N N}\right)}{5 M_{N}}$ \\
$g_{\sigma}=\frac{1}{3} g_{\sigma N N}$ & $g_{\pi}=-\frac{\sqrt{2} m_{\Xi_{c c}} g_{\pi N N}}{5 m_{N}}$ & $h_{v}=\sqrt{2} g_{\rho N N}$ & $h_{v}+f_{v}=-\frac{\sqrt{2}}{5} \frac{m_{\Xi_{c c}}}{m_{N}}\left(g_{\rho N N}+f_{\rho N N}\right)$ \\
$m_{\sigma}=600$ & $m_{\pi}=137.27$ & $m_{\eta}=547.85$ & $m_{\rho}=775.49$ \\
$m_{\omega}=782.65$ & $m_{N}=938.27$ & $m_{\Xi_{c c}}=3621.4$ & $m_{\Lambda_{c}}=2286.46$ \\
$m_{\Xi_{c}}=2469.34$ & $m_{\Sigma_{c}}=2453.54$ & $m_{\Xi_{c}^{\prime}}=2576.75$ & $m_{\Sigma_{c}^{*}}=2518.07, m_{\Xi_{c}^{*}}=2645.9$ \\
\hline \hline
\end{tabular}

$$
\begin{aligned}
\Xi_{c c} \mathcal{B}: J^{P} & =0^{+}\left|{ }^{1} S_{0}\right\rangle, \\
J^{P} & =1^{+}\left|{ }^{3} S_{1}\right\rangle,\left|{ }^{3} D_{1}\right\rangle, \\
\Xi_{c c} \mathcal{B}^{*}: J^{P} & =1^{+}\left|{ }^{3} S_{1}\right\rangle,\left|{ }^{3} D_{1}\right\rangle,\left|{ }^{5} D_{1}\right\rangle, \\
J^{P} & =2^{+}\left|{ }^{5} S_{2}\right\rangle,\left|{ }^{3} D_{2}\right\rangle,\left|{ }^{5} D_{2}\right\rangle
\end{aligned}
$$

with

$$
\begin{aligned}
\left.\left.\Xi_{c c} \mathcal{B}\right|^{2 S+1} L_{J}\right\rangle & =\sum_{m, n, m_{L}} C_{\frac{1}{2}, m ; \frac{1}{2}, n}^{S, m s} C_{S, m_{S} ; L, m_{L}}^{J, m_{J}} \chi_{\frac{1}{2}, m} \chi_{\frac{1}{2}, n} Y_{L, m_{L}}, \\
\left.\left.\Xi_{c c} \mathcal{B}^{*}\right|^{2 S+1} L_{J}\right\rangle & =\sum_{m, n, m_{L}} C_{\frac{1}{2}, m, \frac{3}{2}, n}^{S, m_{S}} C_{S, m_{S} ; L, m_{L}}^{J, m_{J}} \chi_{\frac{1}{2}, m} \Phi_{\frac{3}{2}, n} Y_{L, m_{L}}
\end{aligned}
$$

Here, $C_{\frac{1}{2}, m: \frac{1}{2}, n}^{S, m s}, C_{\frac{1}{2}, m ; \frac{3}{2}, n}^{S, m_{S}}$, and $C_{S, m_{S} ; L, m_{L}}^{J, m_{J}}$ are the ClebschGordan coefficients. $\chi_{\frac{1}{2}, m}$ and $\Phi_{\frac{3}{2}, n}$ are defined as the spin wave functions for baryons with spin $1 / 2$ and spin $3 / 2$, respectively. And $\Phi_{\frac{3}{2}, n}=\sum_{n_{1}, n_{2}}\left\langle\frac{1}{2}, n_{1} ; 1, n_{2} \mid \frac{3}{2}, n\right\rangle \chi_{\frac{1}{2}, n_{1}} \epsilon^{n_{2}}$. The polarization vector $\epsilon$ has the form of $\epsilon_{ \pm}^{m}=\mp \frac{1}{\sqrt{2}} \times$ $\left(\epsilon_{x}^{m} \pm i \epsilon_{y}^{m}\right) \quad$ and $\quad \epsilon_{0}^{m}=\epsilon_{z}^{m} \quad$ with $\quad \epsilon_{ \pm 1}=\frac{1}{\sqrt{2}}(0, \pm 1, i, 0)$ and $\epsilon_{0}=(0,0,0,-1)$.

\section{NUMERICAL RESULTS}

After getting all of the effective potentials for these discussed systems composed of a $S$-wave doubly charmed baryon and an $S$-wave charmed baryon (see Appendix for more details), we solve the coupled channel Schrödinger equation and try to find the corresponding bound state solutions (binding energy $E$ and root-mean-square (RMS) radius $r_{\mathrm{RMS}}$ ).

In the following, we present the results for single- and coupled-channel cases, separately.

\section{A. Single-channel case}

\section{1. $\Xi_{c c} \Lambda_{c}$ and $\Xi_{c c} \Xi_{c}$ systems}

The forbidden $\pi / \eta-\Lambda_{c}-\Lambda_{c}$ and $\pi / \eta-\Xi_{c}-\Xi_{c}$ interactions explain why there don't exist the $\pi / \eta$-exchange potentials for the $\Xi_{c c} \Lambda_{c}$ and $\Xi_{c c} \Xi_{c}$ systems. Additionally, the $\rho$-exchange potential is also absent for the $\Xi_{c c} \Lambda_{c}$ system
TABLE II. Flavor wave functions for the discussed triplecharm hexaquark systems composed of a S-wave double-charm baryon and an $S$-wave charmed baryon.

\begin{tabular}{lll}
\hline \hline Systems & $\left|I, I_{3}\right\rangle$ & \multicolumn{1}{c}{ Configurations } \\
\hline$\Xi_{c c} \Lambda_{c}$ & $\left|\frac{1}{2}, \frac{1}{2}\right\rangle$ & $\left|\Xi_{c c}^{++} \Lambda_{c}^{+}\right\rangle$ \\
& $\left|\frac{1}{2},-\frac{1}{2}\right\rangle$ & $\left|\Xi_{c c}^{+} \Lambda_{c}^{+}\right\rangle$ \\
$\Xi_{c c} \Xi_{c}^{(1, *)}$ & $|1,1\rangle$ & $\left|\Xi_{c c}^{++} \Xi_{c}^{(1, *)+}\right\rangle$ \\
& $|1,0\rangle$ & $\frac{1}{\sqrt{2}}\left(\left|\Xi_{c c}^{++} \Xi_{c}^{(1, *) 0}\right\rangle+\left|\Xi_{c c}^{+} \Xi_{c}^{(1, *)+}\right\rangle\right)$ \\
& $|1,-1\rangle$ & $\left|\Xi_{c c}^{+} \Xi_{c}^{(\prime, *)}\right\rangle$ \\
& $|0,0\rangle$ & $\frac{1}{\sqrt{2}}\left(\left|\Xi_{c c}^{++} \Xi_{c}^{(1, *) 0}\right\rangle-\left|\Xi_{c c}^{+} \Xi_{c}^{(1, *)+}\right\rangle\right)$ \\
$\Xi_{c c} \Sigma_{c}^{(*)}$ & $\left|\frac{1}{2}, \frac{1}{2}\right\rangle$ & $\frac{1}{\sqrt{3}}\left|\Xi_{c c}^{++} \Sigma_{c}^{(*)+}\right\rangle-\sqrt{\frac{2}{3}}\left|\Xi_{c c}^{+} \Sigma_{c}^{(*)++}\right\rangle$ \\
& $\left|\frac{1}{2},-\frac{1}{2}\right\rangle$ & $\sqrt{\frac{2}{3}}\left|\Xi_{c c}^{++} \Sigma_{c}^{(*) 0}\right\rangle-\frac{1}{\sqrt{3}}\left|\Xi_{c c}^{+} \Sigma_{c}^{(*)+}\right\rangle$ \\
& $\left|\frac{3}{2}, \frac{3}{2}\right\rangle$ & $\left|\Xi_{c c}^{++} \Sigma_{c}^{(*)++}\right\rangle$ \\
& $\left|\frac{3}{2}, \frac{1}{2}\right\rangle$ & $\sqrt{\frac{2}{3}}\left|\Xi_{c c}^{++} \Sigma_{c}^{+(*)}\right\rangle+\frac{1}{\sqrt{3}}\left|\Xi_{c c}^{+} \Sigma_{c}^{(*)++}\right\rangle$ \\
& $\left|\frac{3}{2},-\frac{1}{2}\right\rangle$ & $\frac{1}{\sqrt{3}}\left|\Xi_{c c}^{++} \Sigma_{c}^{(*) 0}\right\rangle+\sqrt{\frac{2}{3}}\left|\Xi_{c c}^{+} \Sigma_{c}^{(*)+}\right\rangle$ \\
& $\left|\frac{3}{2},-\frac{3}{2}\right\rangle$ & $\left|\Xi_{c c}^{+} \Sigma_{c}^{(*) 0}\right\rangle$ \\
\hline \hline
\end{tabular}

since it is forbidden by the isospin symmetry. The properties for the OBE effective potentials from other remaining exchanged mesons have been constructed in Refs. [23,24]. The interactions from the $\sigma, \omega$, and $\rho$ exchanges depend on the quark configurations and the isospin of the concrete hadron-hadron systems. Here, the quark configuration for the $\Xi_{c c} \Lambda_{c}$ and $\Xi_{c c} \Xi_{c}$ systems is $(c c q)-(c q q)$ with $q=u$, $d, s$. Thus, the $\sigma$ exchange always provides an attractive force, and the $\omega$ exchange has contribution to repulsive potential. Because the $\rho$ couples to the isospin charge, the effective potential from the $\rho$ exchange is strongly attractive for the isoscalar $\Xi_{c c} \Xi_{c}$ system and weakly repulsive for the isovector $\Xi_{c c} \Xi_{c}$ system. In Fig. 2, we present the $r$ dependence of the effective potentials for the $\Xi_{c c} \Lambda_{c}$ system with $I\left(J^{P}\right)=1 / 2\left(0^{+}\right)$and the $\Xi_{c c} \Xi_{c}$ system with $I\left(J^{P}\right)=0\left(0^{+}\right)$, where the cutoff $\Lambda$ is taken as $1 \mathrm{GeV}$.

By tuning cutoff value $\Lambda$ from 0.8 to $5 \mathrm{GeV}$, we cannot find bound solutions for the $\Xi_{c c} \Lambda_{c}$ systems and the isovector $\Xi_{c c} \Xi_{c}$ systems. In Fig. 3, we present the corresponding bound state properties for the isoscalar $\Xi_{c c} \Xi_{c}$ 


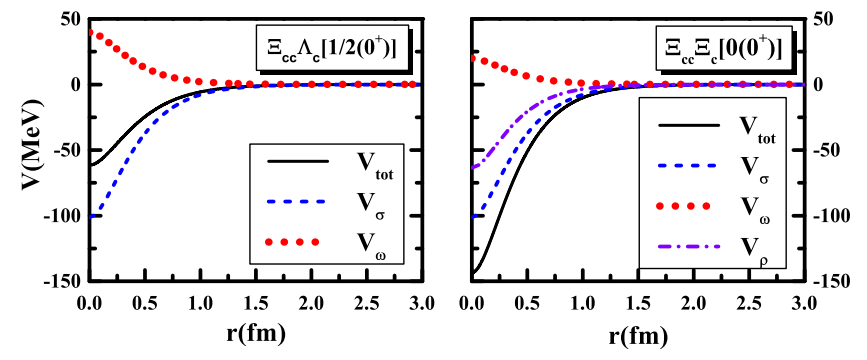

FIG. 2. The $r$ dependence of the deduced effective potentials for the $\Xi_{c c} \Lambda_{c}$ system with $I\left(J^{P}\right)=1 / 2\left(0^{+}\right)$and the $\Xi_{c c} \Xi_{c}$ system with $I\left(J^{P}\right)=0\left(0^{+}\right)$. Here, we take the cutoff $\Lambda=1.00 \mathrm{GeV}$.
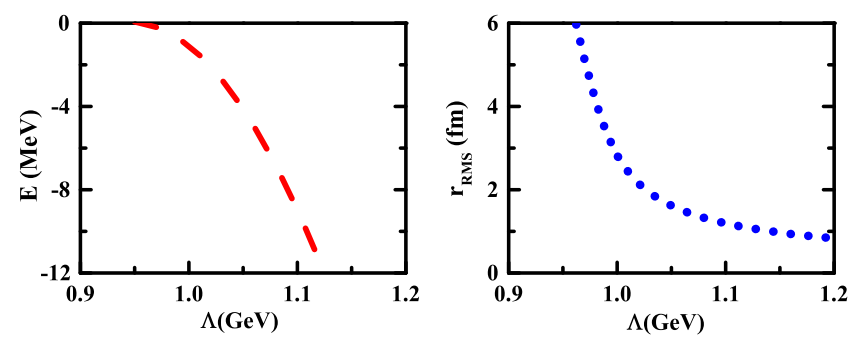

FIG. 3. Bound state solutions (binding energy $E$ and RMS radius $\left.r_{\mathrm{RMS}}\right)$ for the $\Xi_{c c} \Xi_{c}$ system with $I\left(J^{P}\right)=0\left(0^{+} / 1^{+}\right)$.

systems. Here, when cutoff $\Lambda$ is taken around $1 \mathrm{GeV}$, binding energy of several $\mathrm{MeV}$ is obtained, and the RMS radius is larger than $1 \mathrm{fm}$, which is consistent with a typical size of a hadron-hadron molecular state. Thus, there is the possibility that the isoscalar $\Xi_{c c} \Xi_{c}$ states with $J^{P}=0^{+}, 1^{+}$ can be good candidates of triple-charm molecular hexaquarks, depending on the actual value of the cutoff $\Lambda$.

The suggested decay channels of the predicted isoscalar $\Xi_{c c} \Xi_{c}$ states with $J^{P}=0^{+}, 1^{+}$are $\Omega_{c c} \Lambda_{c}$ and $\Omega_{c c c} \Lambda$. Due to the absence of $\Omega_{c c c}$ in experiment, there still exists big challenge for searching for these predicted triple-charm molecular hexaquarks.

\section{2. $\Xi_{c c} \Sigma_{c}^{(*)}$ and $\Xi_{c c} \Xi_{c}^{(\prime, *)}$ systems}

For the $\Xi_{c c} \Sigma_{c}^{(*)}$ and $\Xi_{c c} \Xi_{c}^{(\prime, *)}$ systems, the $\pi$ exchange is allowed, which plays an important role to the $\Xi_{c c} \Sigma_{c}^{(*)}$ and $\Xi_{c c} \Xi_{c}^{(1, *)}$ molecular systems.

In Fig. 4, we present the $\Lambda$ dependence of the binding energies of these $\Xi_{c c} \Sigma_{c}^{(*)}$ and $\Xi_{c c} \Xi_{c}^{(\prime, *)}$ systems, which shows that there exist bound state solutions for the $\Xi_{c c} \Sigma_{c}^{(*)}$ and $\Xi_{c c} \Xi_{c}^{(\prime, *)}$ systems with all allowed isospin and spinparity quantum numbers. Our results suggest that several systems may be possible candidates of triple-charm molecular hexaquarks, which are the $\Xi_{c c} \Sigma_{c}$ states with $I\left(J^{P}\right)=1 / 2\left(0^{+}, 1^{+}\right), 3 / 2\left(0^{+}\right)$, the $\Xi_{c c} \Sigma_{c}^{*}$ states with $I\left(J^{P}\right)=1 / 2\left(2^{+}, 1^{+}\right), 3 / 2\left(2^{+}\right)$, the $\Xi_{c c} \Xi_{c}^{\prime}$ states with
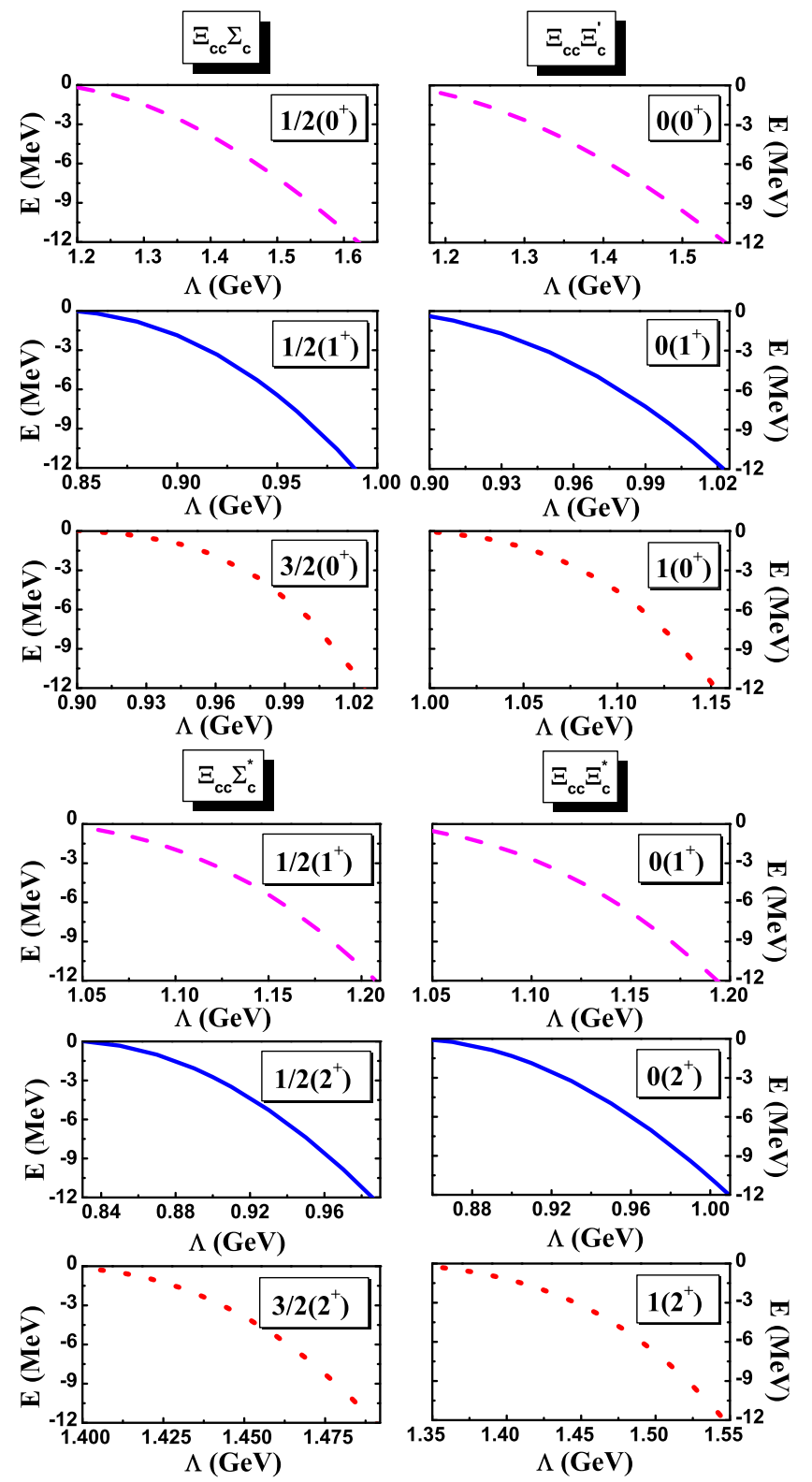

FIG. 4. $\Lambda$ dependence of binding energy $E$ for the $\Xi_{c c} \Sigma_{c}^{(*)}$ and $\Xi_{c c} \Xi_{c}^{(\prime, *)}$ systems.

$I\left(J^{P}\right)=0\left(0^{+}, 1^{+}\right), 1\left(0^{+}\right)$, and the $\Xi_{c c} \Xi_{c}^{*}$ states with $I\left(J^{P}\right)=0\left(1^{+}, 2^{+}\right), 1\left(2^{+}\right)$.

For the $\Xi_{c c} \Sigma_{c}^{(*)}$ systems with $I\left(J^{P}\right)=3 / 2\left(1^{+}\right)$and the $\Xi_{c c} \Xi_{c}^{(\prime, *)}$ systems with $I\left(J^{P}\right)=1\left(1^{+}\right)$, when cutoff is tuned from 2 to $3 \mathrm{GeV}$, we can obtain a binding energy around a few to $10 \mathrm{MeV}$.

For providing more abundant information of experimental search for them, in the following, we further discuss their decay behaviors. Possible two-body strong decay channels are $\Xi_{c c} \Lambda_{c}, \Xi_{c c} \Sigma_{c}, \Omega_{c c c} N$, and $\Omega_{c c c} \Delta$ for the predicted $\Xi_{c c} \Sigma_{c}^{(*)}$ molecular states. For the $\Xi_{c c} \Xi_{c}^{(\prime, *)}$ molecular states, their allowed decay modes include the $\Xi_{c c} \Xi_{c}^{(\prime)}, \Omega_{c c c} \Lambda$, and $\Omega_{c c c} \Sigma$ channels. 
TABLE III. Possible channels involved in the coupled-channel investigation.

\begin{tabular}{llll}
\hline \hline$I\left(J^{P}\right)$ & \multicolumn{3}{c}{ Channels } \\
\hline $1 / 2\left(0^{+}\right)$ & $\Xi_{c c} \Lambda_{c}\left|{ }^{1} S_{0}\right\rangle$ & $\Xi_{c c} \Sigma_{c}\left|{ }^{1} S_{0}\right\rangle$ & $\Xi_{c c} \Sigma_{c}^{*}\left|{ }^{5} D_{0}\right\rangle$ \\
$1 / 2\left(1^{+}\right)$ & $\Xi_{c c} \Lambda_{c}\left|{ }^{3} S_{1},{ }^{3} D_{1}\right\rangle$ & $\Xi_{c c} \Sigma_{c}\left|{ }^{3} S_{1},{ }^{3} D_{1}\right\rangle$ & $\Xi_{c c} \Sigma_{c}^{*}\left|{ }^{3} S_{1},{ }^{3} D_{1},{ }^{5} D_{1}\right\rangle$ \\
$3 / 2\left(0^{+}\right)$ & $\ldots$ & $\Xi_{c c} \Sigma_{c}\left|{ }^{1} S_{0}\right\rangle$ & $\Xi_{c c} \Sigma_{c}^{*}\left|{ }^{5} D_{0}\right\rangle$ \\
$3 / 2\left(1^{+}\right)$ & $\ldots$ & $\Xi_{c c} \Sigma_{c}\left|{ }^{3} S_{1},{ }^{3} D_{1}\right\rangle$ & $\Xi_{c c} \Sigma_{c}^{*}\left|{ }^{3} S_{1},{ }^{3} D_{1},{ }^{5} D_{1}\right\rangle$ \\
$0,1\left(0^{+}\right)$ & $\Xi_{c c} \Xi_{c}\left|{ }^{1} S_{0}\right\rangle$ & $\Xi_{c c} \Xi_{c}^{\prime}\left|{ }^{1} S_{0}\right\rangle$ & $\Xi_{c c} \Xi_{c}^{*}\left|{ }^{5} D_{0}\right\rangle$ \\
$0,1\left(1^{+}\right)$ & $\Xi_{c c} \Xi_{c}\left|{ }^{3} S_{1},{ }^{3} D_{1}\right\rangle$ & $\Xi_{c c} \Xi_{c}^{\prime}\left|{ }^{3} S_{1},{ }^{3} D_{1}\right\rangle$ & $\Xi_{c c} \Xi_{c}^{*}\left|{ }^{3} S_{1},{ }^{3} D_{1},{ }^{5} D_{1}\right\rangle$ \\
\hline \hline
\end{tabular}

\section{B. Coupled-channel case}

For the study of coupled channels, necessary channels are summarized in Table III. For the isosopin $I=3 / 2$ case,

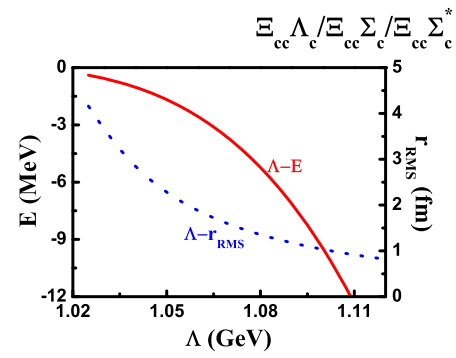

\section{$\left[\mathrm{I}\left(\mathbf{J}^{\mathrm{P}}\right)=\mathbf{1 / 2}\left(\mathbf{0}^{+}\right)\right]$}
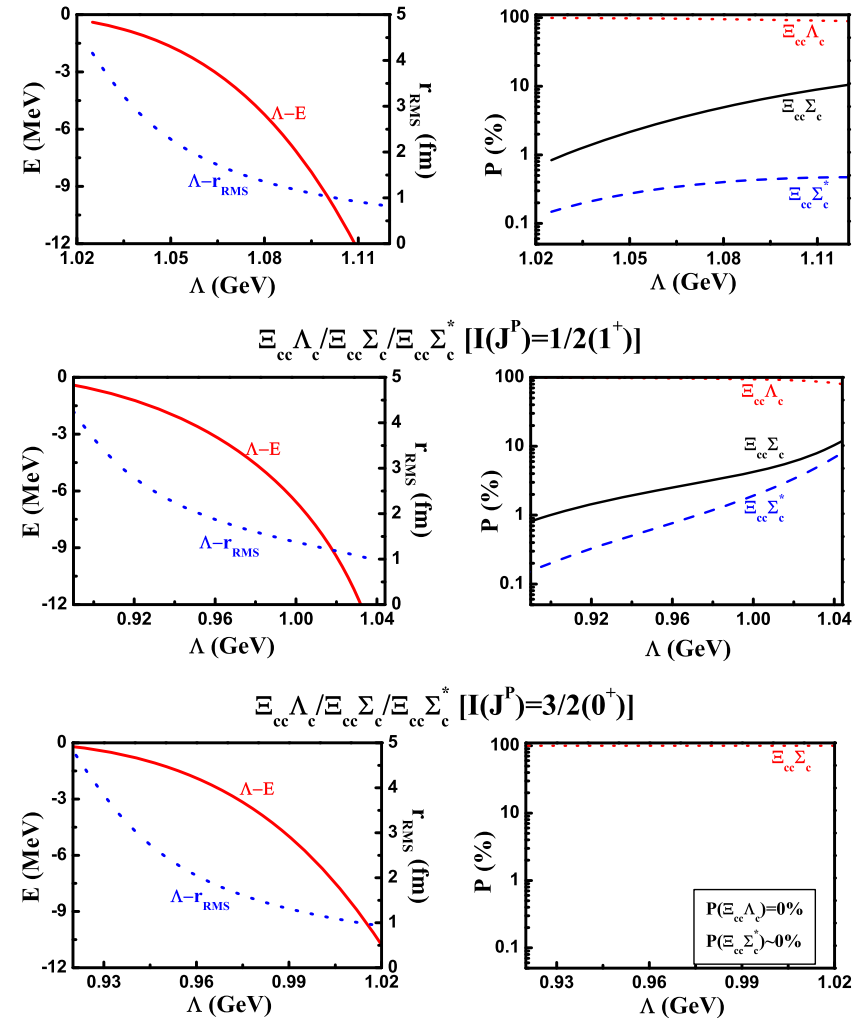

$\Xi_{c c} \Lambda_{c} / \Xi_{c c} \sum_{c} / \Xi_{c c} \Sigma_{c}^{*}\left[I\left(J^{P}\right)=3 / 2\left(1^{+}\right)\right]$
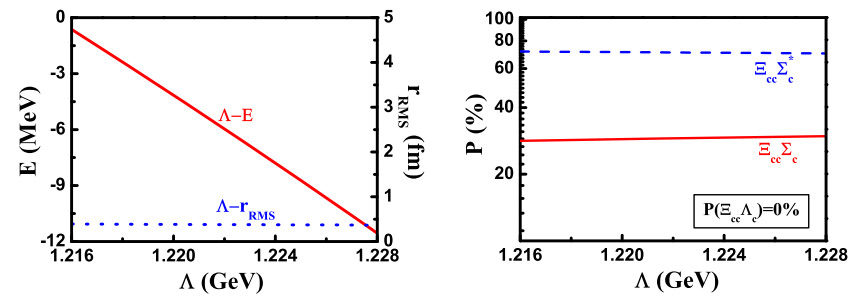

FIG. 5. Left: Bound state properties (binding energy $E$ and root-mean-square radius $r_{\mathrm{RMS}}$ ) for all of the $\Xi_{c c} \Lambda_{c} / \Xi_{c c} \Sigma_{c} / \Xi_{c c} \Sigma_{c}^{*}$ systems when the coupled channel effect is included in our calculation. Right: Probability for the different channels. there are only two systems $\left(\Xi_{c c} \Sigma_{c}\right.$ and $\left.\Xi_{c c} \Sigma_{c}^{*}\right)$ to be considered here.

In Fig. 5, we present the bound state solutions for the investigated $\Xi_{c c} \Lambda_{c} / \Xi_{c c} \Sigma_{c} / \Xi_{c c} \Sigma_{c}^{*}$ systems when considering the coupled-channel effect, where the cutoff $\Lambda$ is taken to be around $1 \mathrm{GeV}$. The results for the $\Xi_{c c} \Lambda_{c} / \Xi_{c c} \Sigma_{c} / \Xi_{c c} \Sigma_{c}^{*}$ coupled systems with $I\left(J^{P}\right)=$ $1 / 2\left(0^{+}\right), 1 / 2\left(1^{+}\right), 3 / 2\left(0^{+}\right)$, and $3 / 2\left(1^{+}\right)$quantum numbers are obtained.

For the $\Xi_{c c} \Lambda_{c} / \Xi_{c c} \Sigma_{c} / \Xi_{c c} \Sigma_{c}^{*}$ coupled system with $I\left(J^{P}\right)=1 / 2\left(0^{+}, 1^{+}\right)$, according to the selected cutoff value $(\Lambda \sim 1 \mathrm{GeV})$ and the obtained RMS radius $\left(r_{\mathrm{RMS}}>1 \mathrm{fm}\right)$, we find that the present meson-exchange approach support both of them may be the loosely bound molecular candidates. Because the dominant
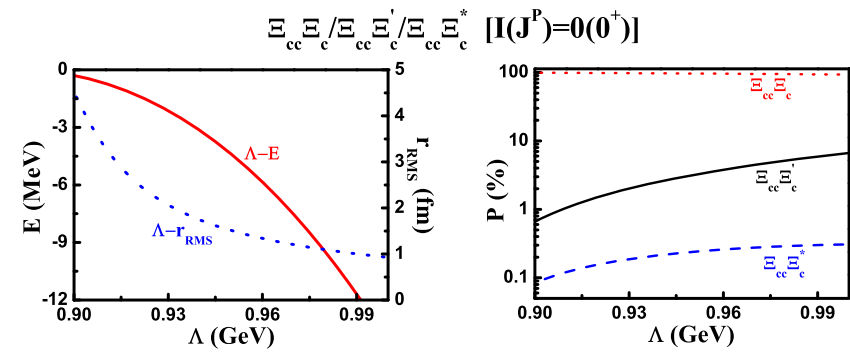

$\Xi_{\mathrm{cc}} \Xi_{\mathrm{c}} / \Xi_{\mathrm{cc}} \Xi_{\mathrm{c}}^{\prime} / \Xi_{\mathrm{cc}} \Xi_{\mathrm{c}}^{*}\left[\mathrm{I}\left(\mathrm{J}^{\mathrm{P}}\right)=0\left(1^{+}\right)\right]$
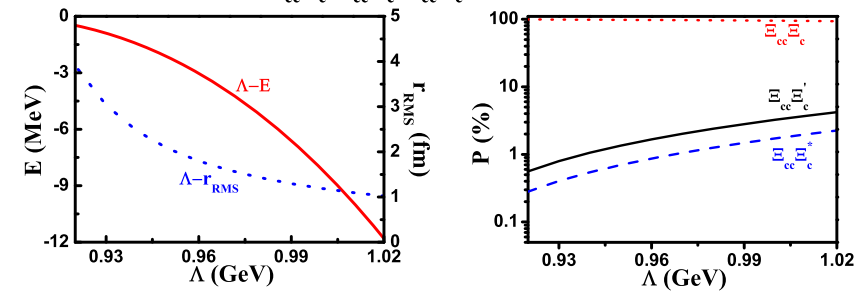

$\Xi_{\mathrm{cc}} \Xi_{c} / \Xi_{\mathrm{cc}} \Xi_{\mathrm{c}}^{\prime} / \Xi_{\mathrm{cc}} \Xi_{\mathrm{c}}^{*}\left[\mathbf{I}\left(\mathrm{J}^{\mathrm{P}}\right)=\mathbf{1}\left(\mathbf{0}^{+}\right)\right]$
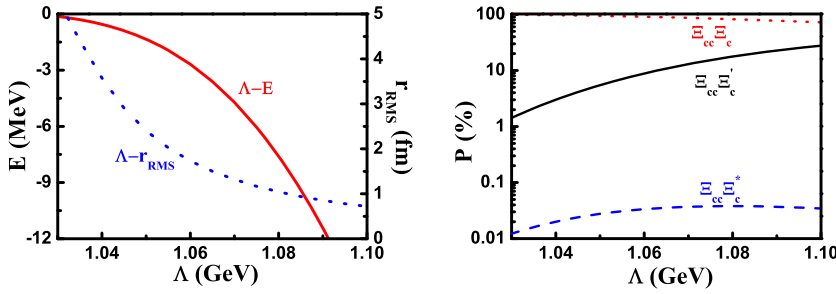

$\Xi_{c c} \Xi_{c} / \Xi_{c c} \Xi_{c}^{\prime} / \Xi_{c c} \Xi_{c}^{*}\left[I\left(J^{P}\right)=1\left(1^{+}\right)\right]$
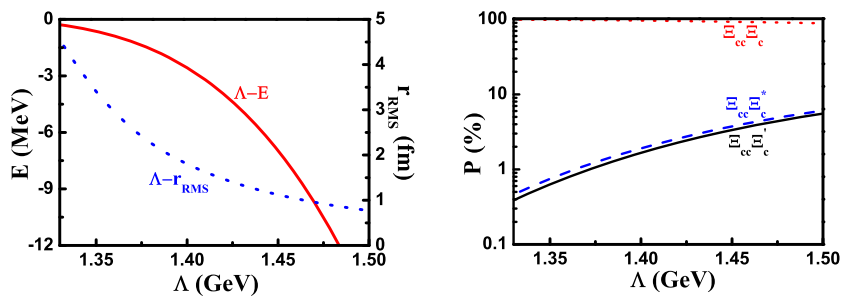

FIG. 6. Left: Bound state properties (binding energy $E$ and root-mean-square radius $r_{\mathrm{RMS}}$ ) for all of the $\Xi_{c c} \Xi_{c} / \Xi_{c c} \Xi_{c}^{\prime} / \Xi_{c c} \Xi_{c}^{*}$ systems when the coupled channel effect is included in our calculation. Right: Probability for the different channels. 
channel is the $\Xi_{c c} \Lambda_{c}$ system with probabilities over $90 \%$, they are mainly composed of the $\Xi_{c c} \Lambda_{c}$ system. Compared to the results for the single $\Xi_{c c} \Lambda_{c}$ system shown in Sec. III A 1, here, we find that the coupled-channel effect plays a very important role to generate these two loosely molecular states. However, for the $\Xi_{c c} \Lambda_{c} / \Xi_{c c} \Sigma_{c} / \Xi_{c c} \Sigma_{c}^{*}$ coupled system with $I\left(J^{P}\right)=3 / 2\left(0^{+}\right)$, our study shows that the coupled-channel effect is not very important. In fact, the bound state solutions do not change very much from the results for the single $\Xi_{c c} \Sigma_{c}$ channel with $I\left(J^{P}\right)=3 / 2\left(0^{+}\right)$, and the probability for the $\Xi_{c c} \Sigma_{c}$ channel is almost $100 \%$ as presented in Fig. 5.

For the $\Xi_{c c} \Lambda_{c} / \Xi_{c c} \Sigma_{c} / \Xi_{c c} \Sigma_{c}^{*}$ system with $I\left(J^{P}\right)=$ $3 / 2\left(1^{+}\right)$shown in Fig. 5, our result indicates that its RMS radius is as small as $0.37 \mathrm{fm}$, and the dominant channel is the $\Xi_{c c} \Sigma_{c}^{*}$ channel with a probability around $70 \%$. It seems that the $\Xi_{c c} \Lambda_{c} / \Xi_{c c} \Sigma_{c} / \Xi_{c c} \Sigma_{c}^{*}$ system with $I\left(J^{P}\right)=3 / 2\left(1^{+}\right)$cannot be a reasonable loosely bound state but a deeply bound state.

In the following, we discuss the $\Xi_{c c} \Xi_{c} / \Xi_{c c} \Xi_{c}^{\prime} / \Xi_{c c} \Xi_{c}^{*}$ coupled-channel systems, as the relevant bound properties are presented in Fig. 6 . For the $I\left(J^{P}\right)=0\left(0^{+}, 1^{+}\right)$case, we also find that the contribution of the coupled-channel effect is not obvious since the obtained property of this coupledchannel system is similar to that of the single $\Xi_{c c} \Xi_{c}$ states with $I\left(J^{P}\right)=0\left(0^{+}, 1^{+}\right)$. However, for the isovector $\Xi_{c c} \Xi_{c} / \Xi_{c c} \Xi_{c}^{\prime} / \Xi_{c c} \Xi_{c}^{*}$ systems, after considering the coupled-channel effect, their bound state properties are obtained, which means that there may exist isovector $\Xi_{c c} \Xi_{c} / \Xi_{c c} \Xi_{c}^{\prime} / \Xi_{c c} \Xi_{c}^{*}$ molecular states with $J^{P}=0^{+}$ and $1^{+}$.

To summarize, after considering the coupled-channel effect, we may predict the existence of four molecular hexaquarks, which are the $\Xi_{c c} \Lambda_{c} / \Xi_{c c} \Sigma_{c} / \Xi_{c c} \Sigma_{c}^{*}$ coupledchannel system with $I\left(J^{P}\right)=1 / 2\left(0^{+}, 1^{+}\right)$, where the $\Xi_{c c} \Lambda_{c}$ channel is dominant, and the $\Xi_{c c} \Xi_{c} / \Xi_{c c} \Xi_{c}^{\prime} / \Xi_{c c} \Xi_{c}^{*}$ coupled systems with $I\left(J^{P}\right)=1\left(0^{+}, 1^{+}\right)$, which mainly couple with the $\Xi_{c c} \Xi_{c}$ channel.

\section{Predictions of other molecular hexaquarks}

In this section, we will extend our study to the interaction between a doubly charmed baryon and an $S$-wave anticharmed baryon. Their OBE effective potentials can be related to the effective potentials for the $\Xi_{c c} \mathcal{B}^{(*)}$ systems by a $G$-parity rule [25], i.e.,

$$
\mathcal{V}^{A \bar{B} \rightarrow A \bar{B}}=\sum_{E} G_{E} \mathcal{V}_{E}^{A B \rightarrow A B}
$$

where $G_{E}$ stands for the $G$-parity for the exchanged meson $E$.

With these obtained effective potentials, we get the corresponding results as presented in Table IV.

If setting the cutoff to be around $1 \mathrm{GeV}$, we can find bound state solutions for all of the discussed systems composed of a doubly charmed baryon and an $S$-wave anticharmed baryon. We find their RMS radius are around $1 \mathrm{fm}$, which is a typical size of the molecular state with small binding energy. These predicted molecular states have typical quark configuration $c c \bar{c} \bar{q} \bar{q} q$. Here, we also list the possible allowed decay channels, i.e., $D D \bar{D}$, $J / \psi D \pi(\eta), D_{s} D \bar{D}, J / \psi D_{s} \pi(\eta), J / \psi D K$, which provide valuable information to further experimental exploration to them.

TABLE IV. Bound state properties (binding energy $E$ and root-mean-square radius $r_{\mathrm{RMS}}$ ) for the molecular hexaquarks composed of a doubly charmed baryon and an $S$-wave anticharmed baryon. Here, $E, r_{\mathrm{RMS}}$, and $\Lambda$ are in units of $\mathrm{MeV}$, fm, and GeV, respectively.

\begin{tabular}{|c|c|c|c|c|c|c|c|c|c|c|c|c|c|c|c|}
\hline$I\left(J^{P}\right)$ & $\Lambda$ & $E$ & $r_{\text {RMS }}$ & $I\left(J^{P}\right)$ & $\Lambda$ & $E$ & $r_{\mathrm{RMS}}$ & $I\left(J^{P}\right)$ & $\Lambda$ & $E$ & $r_{\text {RMS }}$ & $I\left(J^{P}\right)$ & $\Lambda$ & $E$ & $r_{\mathrm{RMS}}$ \\
\hline$\Xi_{c c} \bar{\Lambda}_{c}$ & & & & & & & & $\Xi_{c c} \bar{\Xi}_{c}$ & & & & & & & \\
\hline \multirow[t]{3}{*}{$1 / 2\left(0^{+} / 1^{+}\right)$} & 1.00 & -0.48 & 4.00 & & & & & $0\left(0^{+} / 1^{+}\right)$ & 0.95 & -0.78 & 3.26 & $1\left(0^{+} / 1^{+}\right)$ & 1.10 & -0.39 & 4.20 \\
\hline & 1.10 & -6.77 & 1.35 & & & & & & 1.00 & -5.09 & 1.50 & & 1.30 & -6.20 & 1.36 \\
\hline & 1.20 & -19.44 & 0.90 & & & & & & 1.05 & -13.06 & 1.04 & & $1.50-$ & -16.50 & 0.92 \\
\hline$\Xi_{c c} \bar{\Sigma}_{c}$ & & & & $\Xi_{c c} \bar{\Sigma}_{c}^{*}$ & & & & $\Xi_{c c} \bar{\Xi}_{c}^{\prime}$ & & & & $\Xi_{c c} \bar{\Xi}_{c}^{*}$ & & & \\
\hline \multirow[t]{3}{*}{$1 / 2\left(0^{+}\right)$} & 0.80 & -0.84 & 3.01 & $1 / 2\left(1^{+}\right)$ & 0.80 & -0.41 & 4.01 & $0\left(0^{+}\right)$ & 0.85 & -0.95 & 2.87 & $0\left(1^{+}\right)$ & 0.85 & -1.08 & 2.72 \\
\hline & 0.95 & -7.46 & 1.27 & & 0.84 & -3.86 & 1.60 & & 0.95 & -8.72 & 1.20 & & 0.90 & -7.14 & 1.24 \\
\hline & 1.10 & -10.97 & 1.18 & & 0.88 & -12.52 & 1.01 & & 1.05 & -19.52 & 0.93 & & $0.95-$ & -19.73 & 0.84 \\
\hline \multirow[t]{3}{*}{$1 / 2\left(1^{+}\right)$} & 0.92 & -0.57 & 3.76 & $1 / 2\left(2^{+}\right)$ & 0.95 & -0.14 & 5.60 & $0\left(1^{+}\right)$ & 0.95 & -0.78 & 3.27 & $0\left(2^{+}\right)$ & 1.00 & -0.64 & 3.60 \\
\hline & 0.96 & -4.74 & 1.59 & & 1.05 & -4.93 & 1.65 & & 1.00 & -5.78 & 1.44 & & 1.50 & -6.90 & 1.31 \\
\hline & 1.00 & -13.93 & 1.06 & & 1.15 & -15.19 & 1.12 & & 1.05 & -16.19 & 0.98 & & $1.80-$ & -16.64 & 0.94 \\
\hline \multirow[t]{3}{*}{$3 / 2\left(0^{+}\right)$} & 1.35 & -0.20 & 5.14 & $3 / 2\left(1^{+}\right)$ & 1.00 & -0.59 & 3.57 & $1\left(0^{+}\right)$ & 1.20 & -0.14 & 5.43 & $1\left(1^{+}\right)$ & 1.20 & -0.49 & 3.89 \\
\hline & 1.70 & -4.14 & 1.64 & & 1.55 & -4.40 & 1.60 & & 1.50 & -4.71 & 1.54 & & 1.50 & -4.71 & 1.54 \\
\hline & 2.05 & -9.94 & 1.16 & & 1.85 & -11.84 & 1.08 & & 1.80 & -11.84 & 1.07 & & $1.80-$ & -11.84 & 1.07 \\
\hline \multirow[t]{3}{*}{$3 / 2\left(1^{+}\right)$} & 1.00 & -0.59 & 3.57 & $3 / 2\left(2^{+}\right)$ & 1.00 & -0.71 & 3.32 & $1\left(1^{+}\right)$ & 1.10 & -1.75 & 2.26 & $1\left(2^{+}\right)$ & 1.00 & -0.18 & 5.09 \\
\hline & 1.10 & -4.31 & 1.56 & & 1.10 & -4.42 & 1.54 & & 1.20 & -5.84 & 1.37 & & 1.15 & -5.16 & 1.43 \\
\hline & 1.20 & -11.00 & 1.08 & & 1.20 & -10.73 & 1.08 & & 1.30 & -11.81 & 1.04 & & $1.30-$ & -15.42 & 0.93 \\
\hline
\end{tabular}




\section{SUMMARY}

Exploring the exotic state is an interesting research topic. Especially with the experimental progress on charmoniumumlike $X Y Z$ states and $P_{c}(4380) / P_{c}(4450)$ states, theorists have paid more attentions to the study of exotic states like hidden-charm molecular states and compact multiquarks (see review papers [2,3] for more details).

In 2017, a doubly charmed baryon $\Xi_{c c}^{++}(3621)$ was discovered by LHCb [11]. This new observation makes the study of the interaction between a doubly charmed baryon and an $S$-wave charmed baryon become possible. In this work, we focus on this typical exotic hadronic configuration. By applying the OBE model, we extract their effective potentials, by which we try to find their bound state solutions by solving the Schrödinger equation. This information is crucial to conclude whether there exist the corresponding triple-charm molecular hexaquarks. In the present study, the $S$ - $D$ mixing effect and the coupled-channel effect are taken into account. In this work, all of the coupling constants are determined from the nucleon-nucleon interaction and by using the valence quark structure of charmed baryons. In this regard, the molecular hadrons of doubly charmed hadrons allow us to study the interactions of a single valence quark. Cutoff $\Lambda$ is roughly estimated around $1 \mathrm{GeV}$, which is also widely accepted as a reasonable input from the experience of studying the deuteron in Refs. [21,22].

To clarify the uncertainty of cutoff $\Lambda$, we present the $\Lambda$ dependence of the bound properties for all the possible molecular candidates in the last section. Obviously, it cannot be molecular candidates as binding energies depend very sensitively on the cutoff parameter, like the coupled $\Xi_{c c} \Lambda_{c} / \Xi_{c c} \Sigma_{c} / \Xi_{c c} \Sigma_{c}^{*}$ system with $I\left(J^{P}\right)=3 / 2\left(1^{+}\right)$.

Finally, our discussions are at the qualitative level, we should admit that we cannot make very quantitative predictions. Nevertheless, we imply a serial of possible triple-charm molecular hexaquark states, which are summarized in Table V. When making comparison of the results with and without the coupled-channel effect, we find that the coupled-channel effect plays an essential role for some discussed coupled-channel systems.

As a byproduct, we further extend our study to the interaction between a doubly charmed baryon and an $S$-wave anticharmed baryon since its effective potential can be related to that of the system composed of a doubly charmed baryon and an $S$-wave charmed baryon by $G$-parity rule. Furthermore, we could predict the existence of molecular hexaquarks candidates with typical quark configuration $c c \bar{c} \bar{q} \bar{q} q$. The experimental search for them is also an intriguing issue.

About 15 years ago, the observed $X(3872)$ [26] stimulated extensive discussion of molecular state constructed by charmed meson pair. Later, in 2015, the observation of two hidden-charm $P_{c}$ states [1] was suggested to be a molecular system composed of a charmed meson and a charmed baryon. The reported double-charm baryon $\Xi_{c c}^{++}(3621)$ [11] again provides us a good chance to study the doublecharm baryon interacting with other hadrons. Along this line, we carried out a realistic study of triple-charm molecular hexaquarks and predicted the existence of them. In the next decades, we have reason to believe that the predictions can be accessible at future experiments, which will be full of opportunities and challenges.

\section{ACKNOWLEDGMENTS}

This project is partly supported by the National Natural Science Foundation of China under Grants No. 11222547 and No. 11175073 and by the Fundamental Research Funds for the Central Universities. R. C. is supported by the China Scholarship Council. X. L. is also supported in part by the National Program for Support of Top-notch Young Professionals. A. H. is supported by the JSPS KAKENHI [the Grant-in-Aid for Scientific Research from the Japan Society for the Promotion of Science (JSPS)] with Grant No. JP26400273(C).

\section{APPENDIX: RELEVANT SUBPOTENTIALS}

The exact OBE effective potentials for all of the investigated processes are expressed as

$$
V_{I=1 / 2}^{\Xi_{c c} \Lambda_{c} \rightarrow \Xi_{c c} \Lambda_{c}}=-2 g_{\sigma} l_{B} Y\left(\Lambda, m_{\sigma}, r\right)+\frac{1}{\sqrt{2}} h_{v} \beta_{B} g_{V} Y\left(\Lambda, m_{\omega}, r\right),
$$

$$
\begin{aligned}
\mathcal{V}_{I=1 / 2}^{\Xi_{c c} \Lambda_{c} \rightarrow \Xi_{c c} \Sigma_{c}} & \\
= & -\frac{\sqrt{2}}{12} \frac{g_{\pi} g_{1}}{f_{\pi} m_{\Xi_{c c}}}\left[\mathcal{A}_{1} \mathcal{O}_{r}+\mathcal{A}_{2} \mathcal{P}_{r}\right] Y\left(\Lambda_{0}, m_{\pi 0}, r\right) \\
& -\frac{\lambda_{I} g_{V}\left(h_{V}-f_{V}\right)}{6 m_{\Xi_{c c}}}\left[2 \mathcal{A}_{1} \mathcal{O}_{r}-\mathcal{A}_{2} \mathcal{P}_{r}\right] Y\left(\Lambda_{0}, m_{\rho 0}, r\right) \\
& +\frac{\lambda_{I} g_{V} h_{V}}{m_{\Xi_{c c}}} \mathcal{A}_{3} \mathcal{Q}_{r} Y\left(\Lambda_{0}, m_{\rho 0}, r\right),
\end{aligned}
$$

TABLE V. A summary of predicted hexaquark molecular states.

\begin{tabular}{llll}
\hline \hline States & \multicolumn{1}{c}{$I\left(J^{P}\right)$} & States & \multicolumn{1}{c}{$I\left(J^{P}\right)$} \\
\hline$\Xi_{c c} \Lambda_{c}$ & $1 / 2\left(0^{+}\right), 1 / 2\left(1^{+}\right)$ & $\Xi_{c c} \Xi_{c}$ & $0\left(0^{+}\right), 0\left(1^{+}\right), 1\left(0^{+}\right), 1\left(1^{+}\right)$ \\
$\Xi_{c c} \Sigma_{c}$ & $1 / 2\left(0^{+}\right), 1 / 2\left(1^{+}\right), 3 / 2\left(0^{+}\right)$ & $\Xi_{c c} \Xi_{c}^{\prime}$ & $0\left(0^{+}\right), 0\left(1^{+}\right), 1\left(0^{+}\right)$ \\
$\Xi_{c c} \Sigma_{c}^{*}$ & $1 / 2\left(1^{+}\right), 1 / 2\left(2^{+}\right), 3 / 2\left(2^{+}\right)$ & $\Xi_{c c} \Xi_{c}^{*}$ & $0\left(1^{+}\right), 0\left(2^{+}\right), 1\left(2^{+}\right)$ \\
\hline \hline
\end{tabular}




$$
\begin{aligned}
\mathcal{V}_{I=1 / 2}^{\Xi_{c c} \Lambda_{c} \rightarrow \Xi_{c c} \Sigma_{c}^{*}}= & -\frac{1}{2 \sqrt{6}} \frac{g_{\pi} g_{4}}{f_{\pi} m_{\Xi_{c c}}}\left[\mathcal{A}_{8} \mathcal{O}_{r}+\mathcal{A}_{9} \mathcal{P}_{r}\right] Y\left(\Lambda_{1}, m_{\pi 1}, r\right)+\frac{\sqrt{3}}{2} \frac{\lambda_{I} g_{V} h_{V}}{m_{\Xi_{c c}}} \mathcal{A}_{10} \mathcal{Q}_{r} Y\left(\Lambda_{1}, m_{\rho 1}, r\right) \\
& -\frac{\sqrt{3}}{12} \frac{\lambda_{I} g_{V}\left(h_{V}-f_{V}\right)}{m_{\Xi_{c c}}}\left[2 \mathcal{A}_{8} \mathcal{O}_{r}-\mathcal{A}_{9} \mathcal{P}_{r}\right] Y\left(\Lambda_{1}, m_{\rho 1}, r\right),
\end{aligned}
$$

$$
\begin{aligned}
& V_{I}^{\Xi_{c c} \Sigma_{c} \rightarrow \Xi_{c c} \Sigma_{c}}=g_{\sigma} l_{S} Y\left(\Lambda, m_{\sigma}, r\right)-\frac{\mathcal{H}(I)}{12} \frac{g_{\pi} g_{1}}{f_{\pi} M_{\Xi_{c c}}}\left(\mathcal{A}_{1} \mathcal{O}_{r}+\mathcal{A}_{2} \mathcal{P}_{r}\right) Y\left(\Lambda, m_{\pi}, r\right)-\frac{g_{\pi} g_{1}}{36 f_{\pi} M_{\Xi_{c c}}}\left(\mathcal{A}_{1} \mathcal{O}_{r}+\mathcal{A}_{2} \mathcal{P}_{r}\right) Y\left(\Lambda, m_{\eta}, r\right) \\
& -\frac{\mathcal{H}(I)}{2}\left(\frac{h_{v} \beta_{S} g_{V}}{\sqrt{2}}+\frac{h_{v} \lambda_{S} g_{V}}{3 \sqrt{2} M_{\Sigma_{c}}} \mathcal{O}_{r}\right) Y\left(\Lambda, m_{\rho}, r\right)-\frac{\mathcal{H}(I)}{3 \sqrt{2}} h_{v} \lambda_{S} g_{V}\left(\frac{1}{M_{\Sigma_{c}}}+\frac{1}{M_{\Xi_{c c}}}\right) \mathcal{A}_{3} \mathcal{Q}_{r} Y\left(\Lambda, m_{\rho}, r\right) \\
& -\frac{\mathcal{H}(I)}{18 \sqrt{2}} \frac{\left(f_{v}+h_{v}\right) \lambda_{S} g_{V}}{M_{\Xi_{c c}}}\left(2 \mathcal{A}_{1} \mathcal{O}_{r}-\mathcal{A}_{2} \mathcal{P}_{r}\right) Y\left(\Lambda, m_{\rho}, r\right)-\frac{1}{2}\left(\frac{h_{v} \beta_{S} g_{V}}{\sqrt{2}}+\frac{h_{v} \lambda_{S} g_{V}}{3 \sqrt{2} M_{\Sigma_{c}}} \mathcal{O}_{r}\right) Y\left(\Lambda, m_{\omega}, r\right) \\
& -\frac{1}{3 \sqrt{2}} h_{v} \lambda_{S} g_{V}\left(\frac{1}{M_{\Sigma_{c}}}+\frac{1}{M_{\Xi_{c c}}}\right) \mathcal{A}_{3} \mathcal{Q}_{r} Y\left(\Lambda, m_{\omega}, r\right)-\frac{1}{18 \sqrt{2}} \frac{\left(f_{v}+h_{v}\right) \lambda_{S} g_{V}}{M_{\Xi_{c c}}}\left(2 \mathcal{A}_{1} \mathcal{O}_{r}-\mathcal{A}_{2} \mathcal{P}_{r}\right) Y\left(\Lambda, m_{\omega}, r\right),
\end{aligned}
$$

$$
\begin{aligned}
\mathcal{V}_{I}^{\Xi_{c c} \Sigma_{c} \rightarrow \Xi_{c c} \Sigma_{c}^{*}}= & -\frac{\mathcal{H}(I)}{4 \sqrt{3}} \frac{g_{\pi} g_{1}}{f_{\pi} m_{\Xi_{c c}}}\left[\mathcal{A}_{11} \mathcal{O}_{r}+\mathcal{A}_{12} \mathcal{P}_{r}\right] Y\left(\Lambda_{2}, m_{\pi 2}, r\right)-\frac{1}{24 \sqrt{3}} \frac{g_{\pi} g_{1}}{f_{\pi} m_{\Xi_{c c}}}\left[\mathcal{A}_{11} \mathcal{O}_{r}+\mathcal{A}_{12} \mathcal{P}_{r}\right] Y\left(\Lambda_{2}, m_{\eta 2}, r\right) \\
& +\frac{\mathcal{H}(I)}{\sqrt{6}} \frac{\lambda_{S} g_{V} h_{V}}{m_{\Xi_{c c}}} \mathcal{A}_{13} \mathcal{Q}_{r} Y\left(\Lambda_{2}, m_{\rho 2}, r\right)-\frac{\mathcal{H}(I)}{6 \sqrt{6}} \frac{\lambda_{S} g_{V}\left(f_{V}-h_{V}\right)}{m_{\Xi_{c c}}}\left[2 \mathcal{A}_{11} \mathcal{O}_{r}-\mathcal{A}_{12} \mathcal{P}_{r}\right] Y\left(\Lambda_{2}, m_{\rho 2}, r\right) \\
& +\frac{1}{2 \sqrt{6}} \frac{\lambda_{S} g_{V} h_{V}}{m_{\Xi_{c c}}} \mathcal{A}_{13} \mathcal{Q}_{r} Y\left(\Lambda_{2}, m_{\omega 2}, r\right)-\frac{1}{12 \sqrt{6}} \frac{\lambda_{S} g_{V}\left(f_{V}-h_{V}\right)}{m_{\Xi_{c c}}}\left[2 \mathcal{A}_{11} \mathcal{O}_{r}-\mathcal{A}_{12} \mathcal{P}_{r}\right] Y\left(\Lambda_{2}, m_{\omega 2}, r\right),
\end{aligned}
$$

$$
\begin{aligned}
& V_{I}^{\Xi_{c c} \Sigma_{c}^{*} \rightarrow \Xi_{c c} \Sigma_{c}^{*}}=g_{\sigma} l_{S} \mathcal{A}_{4} Y\left(\Lambda, m_{\sigma}, r\right)-\frac{\mathcal{H}(I)}{8} \frac{g_{\pi} g_{1}}{M_{\Xi_{c c}} f_{\pi}}\left[\mathcal{A}_{5} \mathcal{O}_{r}+\mathcal{A}_{6} \mathcal{P}_{r}\right] Y\left(\Lambda, m_{\pi}, r\right)-\frac{1}{24} \frac{g_{\pi} g_{1}}{M_{\Xi_{c c}} f_{\pi}}\left[\mathcal{A}_{5} \mathcal{O}_{r}+\mathcal{A}_{6} \mathcal{P}_{r}\right] Y\left(\Lambda, m_{\eta}, r\right) \\
& -\frac{\mathcal{H}(I)}{2 \sqrt{2}} h_{v} \beta_{S} g_{V} \mathcal{A}_{4} Y\left(\Lambda, m_{\rho}, r\right)-\frac{\mathcal{H}(I)}{2 \sqrt{2}} \frac{h_{v} \lambda_{S} g_{V}}{M_{\Xi_{c c}}} \mathcal{A}_{7} \mathcal{Q}_{r} Y\left(\Lambda, m_{\rho}, r\right) \\
& +\frac{\mathcal{H}(I)}{12 \sqrt{2}} \frac{\left(h_{v}+f_{v}\right) \lambda_{s} g_{V}}{M_{\Xi_{c c}}}\left[2 \mathcal{A}_{5} \mathcal{O}_{r}-\mathcal{A}_{6} \mathcal{P}_{r}\right] Y\left(\Lambda, m_{\rho}, r\right)-\frac{1}{2 \sqrt{2}} h_{v} \beta_{S} g_{V} \mathcal{A}_{4} Y\left(\Lambda, m_{\omega}, r\right) \\
& -\frac{1}{2 \sqrt{2}} h_{v} \lambda_{S} g_{V} \mathcal{A}_{7} \mathcal{Q}_{r} Y\left(\Lambda, m_{\omega}, r\right)+\frac{1}{12 \sqrt{2}} \frac{\left(h_{v}+f_{v}\right) \lambda_{s} g_{V}}{M_{\Xi_{c c}}}\left[2 \mathcal{A}_{5} \mathcal{O}_{r}-\mathcal{A}_{6} \mathcal{P}_{r}\right] Y\left(\Lambda, m_{\omega}, r\right), \\
& \mathcal{V}_{I}^{\Xi_{c c} \Xi_{c} \rightarrow \Xi_{c c} \Xi_{c}}=-2 g_{\sigma} l_{B} Y\left(\Lambda, m_{\sigma}, r\right)+\frac{\mathcal{G}(I)}{2 \sqrt{2}} h_{v} \beta_{B} g_{V} Y\left(\Lambda, m_{\rho}, r\right)+\frac{1}{2 \sqrt{2}} h_{v} \beta_{B} g_{V} Y\left(\Lambda, m_{\omega}, r\right), \\
& \mathcal{V}_{I}^{\Xi_{c c} \Xi_{c} \rightarrow \Xi_{c c} \Xi_{c}^{\prime}}=-\frac{\mathcal{G}(I)}{12 \sqrt{2}} \frac{g_{\pi} g_{1}}{f_{\pi} m_{\Xi_{c c}}}\left[\mathcal{A}_{1} \mathcal{O}_{r}+\mathcal{A}_{2} \mathcal{P}_{r}\right] Y\left(\Lambda_{3}, m_{\pi 3}, r\right)-\frac{1}{12 \sqrt{2}} \frac{g_{\pi} g_{1}}{f_{\pi} m_{\Xi_{c c}}}\left[\mathcal{A}_{1} \mathcal{O}_{r}+\mathcal{A}_{2} \mathcal{P}_{r}\right] Y\left(\Lambda_{3}, m_{\eta 3}, r\right) \\
& -\frac{\mathcal{G}(I)}{12 \sqrt{3}} \frac{\lambda_{I} g_{V}\left(h_{V}-f_{V}\right)}{m_{\Xi_{c c}}}\left[2 \mathcal{A}_{1} \mathcal{O}_{r}-\mathcal{A}_{2} \mathcal{P}_{r}\right] Y\left(\Lambda_{3}, m_{\rho 3}, r\right)+\frac{\mathcal{G}(I)}{2 \sqrt{3}} \frac{\lambda_{I} g_{V} h_{V}}{m_{\Xi_{c c}}} \mathcal{A}_{3} \mathcal{Q}_{r} Y\left(\Lambda_{3}, m_{\rho 3}, r\right) \\
& -\frac{1}{12 \sqrt{3}} \frac{\lambda_{I} g_{V}\left(h_{V}-f_{V}\right)}{m_{\Xi_{c c}}}\left[2 \mathcal{A}_{1} \mathcal{O}_{r}-\mathcal{A}_{2} \mathcal{P}_{r}\right] Y\left(\Lambda_{3}, m_{\omega 3}, r\right)+\frac{1}{2 \sqrt{3}} \frac{\lambda_{I} g_{V} h_{V}}{m_{\Xi_{c c}}} \mathcal{A}_{3} \mathcal{Q}_{r} Y\left(\Lambda_{3}, m_{\omega 3}, r\right), \\
& \mathcal{V}_{I}^{\Xi_{c c} \Xi_{c} \rightarrow \Xi_{c c} \Xi_{c}^{*}}=-\frac{\mathcal{G}(I)}{12 \sqrt{2}} \frac{g_{\pi} g_{4}}{f_{\pi} m_{\Xi_{c c}}}\left[\mathcal{A}_{8} \mathcal{O}_{r}+\mathcal{A}_{9} \mathcal{P}_{r}\right] Y\left(\Lambda_{4}, m_{\pi 4}, r\right)-\frac{1}{12 \sqrt{2}} \frac{g_{\pi} g_{4}}{f_{\pi} m_{\Xi_{c c}}}\left[\mathcal{A}_{8} \mathcal{O}_{r}+\mathcal{A}_{9} \mathcal{P}_{r}\right] Y\left(\Lambda_{4}, m_{\eta 4}, r\right) \\
& +\frac{\mathcal{G}(I)}{4} \frac{\lambda_{I} g_{V} h_{V}}{m_{\Xi_{c c}}} \mathcal{A}_{10} \mathcal{Q}_{r} Y\left(\Lambda_{4}, m_{\rho 4}, r\right)-\frac{\mathcal{G}(I)}{24} \frac{\lambda_{I} g_{V}\left(h_{V}-f_{V}\right)}{m_{\Xi_{c c}}}\left[2 \mathcal{A}_{8} \mathcal{O}_{r}-\mathcal{A}_{9} \mathcal{P}_{r}\right] Y\left(\Lambda_{4}, m_{\rho 4}, r\right) \\
& +\frac{1}{4} \frac{\lambda_{I} g_{V} h_{V}}{m_{\Xi_{c c}}} \mathcal{A}_{10} \mathcal{Q}_{r} Y\left(\Lambda_{4}, m_{\omega 4}, r\right)-\frac{1}{24} \frac{\lambda_{I} g_{V}\left(h_{V}-f_{V}\right)}{m_{\Xi_{c c}}}\left[2 \mathcal{A}_{8} \mathcal{O}_{r}-\mathcal{A}_{9} \mathcal{P}_{r}\right] Y\left(\Lambda_{4}, m_{\omega 4}, r\right),
\end{aligned}
$$




$$
\begin{aligned}
\mathcal{V}_{I}^{\Xi_{c c} \Xi_{c}^{\prime} \rightarrow \Xi_{c c} \Xi_{c}^{\prime}}= & g_{\sigma} l_{S} Y\left(\Lambda, m_{\sigma}, r\right)-\frac{\mathcal{G}(I)}{24} \frac{g_{\pi} g_{1}}{f_{\pi} M_{\Xi_{c c}}}\left(\mathcal{A}_{1} \mathcal{O}_{r}+\mathcal{A}_{2} \mathcal{P}_{r}\right) Y\left(\Lambda, m_{\pi}, r\right)+\frac{g_{\pi} g_{1}}{72 f_{\pi} M_{\Xi_{c c}}}\left(\mathcal{A}_{1} \mathcal{O}_{r}+\mathcal{A}_{2} \mathcal{P}_{r}\right) Y\left(\Lambda, m_{\eta}, r\right) \\
& -\frac{\mathcal{G}(I)}{4}\left(\frac{h_{v} \beta_{S} g_{V}}{\sqrt{2}}+\frac{h_{v} \lambda_{S} g_{V}}{3 \sqrt{2} M_{\Sigma_{c}}} \mathcal{O}_{r}\right) Y\left(\Lambda, m_{\rho}, r\right)-\frac{\mathcal{G}(I)}{6 \sqrt{2}} h_{v} \lambda_{S} g_{V}\left(\frac{1}{M_{\Sigma_{c}}}+\frac{1}{M_{\Xi_{c c}}}\right) \mathcal{A}_{3} \mathcal{Q}_{r} Y\left(\Lambda, m_{\rho}, r\right) \\
& -\frac{\mathcal{G}(I)}{36 \sqrt{2}} \frac{\left(f_{v}+h_{v}\right) \lambda_{S} g_{V}}{M_{\Xi_{c c}}}\left(2 \mathcal{A}_{1} \mathcal{O}_{r}-\mathcal{A}_{2} \mathcal{P}_{r}\right) Y\left(\Lambda, m_{\rho}, r\right)-\frac{1}{4}\left(\frac{h_{v} \beta_{S} g_{V}}{\sqrt{2}}+\frac{h_{v} \lambda_{S} g_{V}}{3 \sqrt{2} M_{\Sigma_{c}}} \mathcal{O}_{r}\right) Y\left(\Lambda, m_{\omega}, r\right) \\
& -\frac{1}{6 \sqrt{2}} h_{v} \lambda_{S} g_{V}\left(\frac{1}{M_{\Sigma_{c}}}+\frac{1}{M_{\Xi_{c c}}}\right) \mathcal{A}_{3} \mathcal{Q}_{r} Y\left(\Lambda, m_{\omega}, r\right)-\frac{1}{36 \sqrt{2}} \frac{\left(f_{v}+h_{v}\right) \lambda_{S} g_{V}}{M_{\Xi_{c c}}}\left(2 \mathcal{A}_{1} \mathcal{O}_{r}-\mathcal{A}_{2} \mathcal{P}_{r}\right) Y\left(\Lambda, m_{\omega}, r\right),
\end{aligned}
$$

$$
\begin{aligned}
\mathcal{V}_{I}^{\Xi_{c c} \Xi_{c}^{\prime} \rightarrow \Xi_{c c} \Xi_{c}^{*}}= & \frac{\mathcal{G}(I)}{16 \sqrt{3}} \frac{g_{\pi} g_{1}}{f_{\pi} m_{\Xi_{c c}}}\left[\mathcal{A}_{11} \mathcal{O}_{r}+\mathcal{A}_{12} \mathcal{P}_{r}\right] Y\left(\Lambda_{5}, m_{\pi 5}, r\right)+\frac{1}{48 \sqrt{3}} \frac{g_{\pi} g_{1}}{f_{\pi} m_{\Xi_{c c}}}\left[\mathcal{A}_{11} \mathcal{O}_{r}+\mathcal{A}_{12} \mathcal{P}_{r}\right] Y\left(\Lambda_{5}, m_{\eta 5}, r\right) \\
& +\frac{\mathcal{G}(I)}{4 \sqrt{6}} \frac{\lambda_{S} g_{V} h_{V}}{m_{\Xi_{c c}}} \mathcal{A}_{13} \mathcal{Q}_{r} Y\left(\Lambda_{5}, m_{\rho 5}, r\right)-\frac{\mathcal{G}(I)}{24 \sqrt{6}} \frac{\lambda_{S} g_{V}\left(f_{V}-h_{V}\right)}{m_{\Xi_{c c}}}\left[2 \mathcal{A}_{11} \mathcal{O}_{r}-\mathcal{A}_{12} \mathcal{P}_{r}\right] Y\left(\Lambda_{5}, m_{\rho 5}, r\right) \\
& -\frac{1}{4 \sqrt{6}} \frac{\lambda_{S} g_{V} h_{V}}{m_{\Xi_{c c}}} \mathcal{A}_{13} \mathcal{Q}_{r} Y\left(\Lambda_{5}, m_{\omega 5}, r\right)+\frac{1}{24 \sqrt{6}} \frac{\lambda_{S} g_{V}\left(f_{V}-h_{V}\right)}{m_{\Xi_{c c}}}\left[2 \mathcal{A}_{11} \mathcal{O}_{r}-\mathcal{A}_{12} \mathcal{P}_{r}\right] Y\left(\Lambda_{5}, m_{\omega 5}, r\right),
\end{aligned}
$$

$$
\begin{aligned}
\mathcal{V}_{I}^{\Xi_{c c} \Xi_{c}^{*} \rightarrow \Xi_{c c} \Xi_{c}^{*}}= & g_{\sigma} l_{S} \mathcal{A}_{4} Y\left(\Lambda, m_{\sigma}, r\right)-\frac{\mathcal{G}(I)}{16} \frac{g_{\pi} g_{1}}{M_{\Xi_{c c}} f_{\pi}}\left[\mathcal{A}_{5} \mathcal{O}_{r}+\mathcal{A}_{6} \mathcal{P}_{r}\right] Y\left(\Lambda, m_{\pi}, r\right)+\frac{1}{48} \frac{g_{\pi} g_{1}}{M_{\Xi_{c c}} f_{\pi}}\left[\mathcal{A}_{5} \mathcal{O}_{r}+\mathcal{A}_{6} \mathcal{P}_{r}\right] Y\left(\Lambda, m_{\eta}, r\right) \\
& -\frac{\mathcal{G}(I)}{4 \sqrt{2}} h_{v} \beta_{S} g_{V} \mathcal{A}_{4} Y\left(\Lambda, m_{\rho}, r\right)-\frac{\mathcal{G}(I)}{4 \sqrt{2}} \frac{h_{v} \lambda_{S} g_{V}}{M_{\Xi_{c c}}} \mathcal{A}_{7} \mathcal{Q}_{r} Y\left(\Lambda, m_{\rho}, r\right) \\
& +\frac{\mathcal{G}(I)}{24 \sqrt{2}} \frac{\left(h_{v}+f_{v}\right) \lambda_{s} g_{V}}{M_{\Xi_{c c}}}\left[2 \mathcal{A}_{5} \mathcal{O}_{r}-\mathcal{A}_{6} \mathcal{P}_{r}\right] Y\left(\Lambda, m_{\rho}, r\right)-\frac{h_{v} \beta_{S} g_{V}}{4 \sqrt{2}} \mathcal{A}_{4} Y\left(\Lambda, m_{\omega}, r\right) \\
& -\frac{h_{v} \lambda_{S} g_{V}}{4 \sqrt{2}} \mathcal{A}_{7} \mathcal{Q}_{r} Y\left(\Lambda, m_{\omega}, r\right)+\frac{\left(h_{v}+f_{v}\right) \lambda_{s} g_{V}}{24 \sqrt{2} M_{\Xi_{c c}}}\left[2 \mathcal{A}_{5} \mathcal{O}_{r}-\mathcal{A}_{6} \mathcal{P}_{r}\right] Y\left(\Lambda, m_{\omega}, r\right)
\end{aligned}
$$

with

$$
\mathcal{O}_{r}=\frac{1}{r^{2}} \frac{\partial}{\partial r} r^{2} \frac{\partial}{\partial r}, \quad \mathcal{P}_{r}=r \frac{\partial}{\partial r} \frac{1}{r} \frac{\partial}{\partial r}, \quad \mathcal{Q}_{r}=\frac{1}{r} \frac{\partial}{\partial r}, \quad Y(\Lambda, m, r)=\frac{1}{4 \pi r}\left(e^{-m r}-e^{-\Lambda r}\right)-\frac{\Lambda^{2}-m^{2}}{8 \pi \Lambda} e^{-\Lambda r}
$$

The variables in the above effective potentials denote

$$
\begin{aligned}
q_{0} & =\frac{-m_{\Sigma_{c}}^{2}+m_{\Lambda_{c}}^{2}}{2\left(m_{\Xi_{c c}}+m_{\Sigma_{c}}\right)}, \quad q_{1}=\frac{-m_{\Sigma_{c}^{*}}^{2}+m_{\Lambda_{c}}^{2}}{2\left(m_{\Xi_{c c}}+m_{\Sigma_{c}^{*}}\right)}, \quad q_{2}=\frac{-m_{\Sigma_{c}^{*}}^{2}+m_{\Sigma_{c}}^{2}}{2\left(m_{\Xi_{c c}}+m_{\Sigma_{c}^{*}}\right)}, \\
q_{3} & =\frac{-m_{\Xi_{c}^{\prime}}^{2}+m_{\Xi_{c}}^{2}}{2\left(m_{\Xi_{c c}}+m_{\Xi_{c}^{\prime}}\right)}, \quad q_{4}=\frac{-m_{\Xi_{c}^{*}}^{2}+m_{\Xi_{c}}^{2}}{2\left(m_{\Xi_{c c}}+m_{\Xi_{c}^{*}}\right)}, \quad q_{5}=\frac{-m_{\Xi_{c}^{*}}^{2}+m_{\Xi_{c}^{\prime}}^{2}}{2\left(m_{\Xi_{c c}}+m_{\Xi_{c}^{*}}\right)}, \\
\Lambda_{i}^{2} & =\Lambda^{2}-q_{i}^{2}, \quad m_{E i}^{2}=m_{E}^{2}-q_{i}^{2}, \quad i=0,1,2,3,4,5 .
\end{aligned}
$$

Here, we define two isospin factors, $\mathcal{G}(I)$ and $\mathcal{H}(I)$, respectively,

$$
G(I=0)=-3, \quad \mathcal{G}(I=1)=1, \quad \mathcal{H}(I=1 / 2)=-2, \quad \mathcal{H}(I=3 / 2)=1
$$


TABLE VI. Matrix elements for $\langle f|\mathcal{A}| i\rangle$ for the spin-spin, spin-orbit, and tensor force operators $\mathcal{A}$. For example, $\left\langle{ }^{1} S_{0}\left|\mathcal{A}_{1}\right|{ }^{1} S_{0}\right\rangle=-3$.

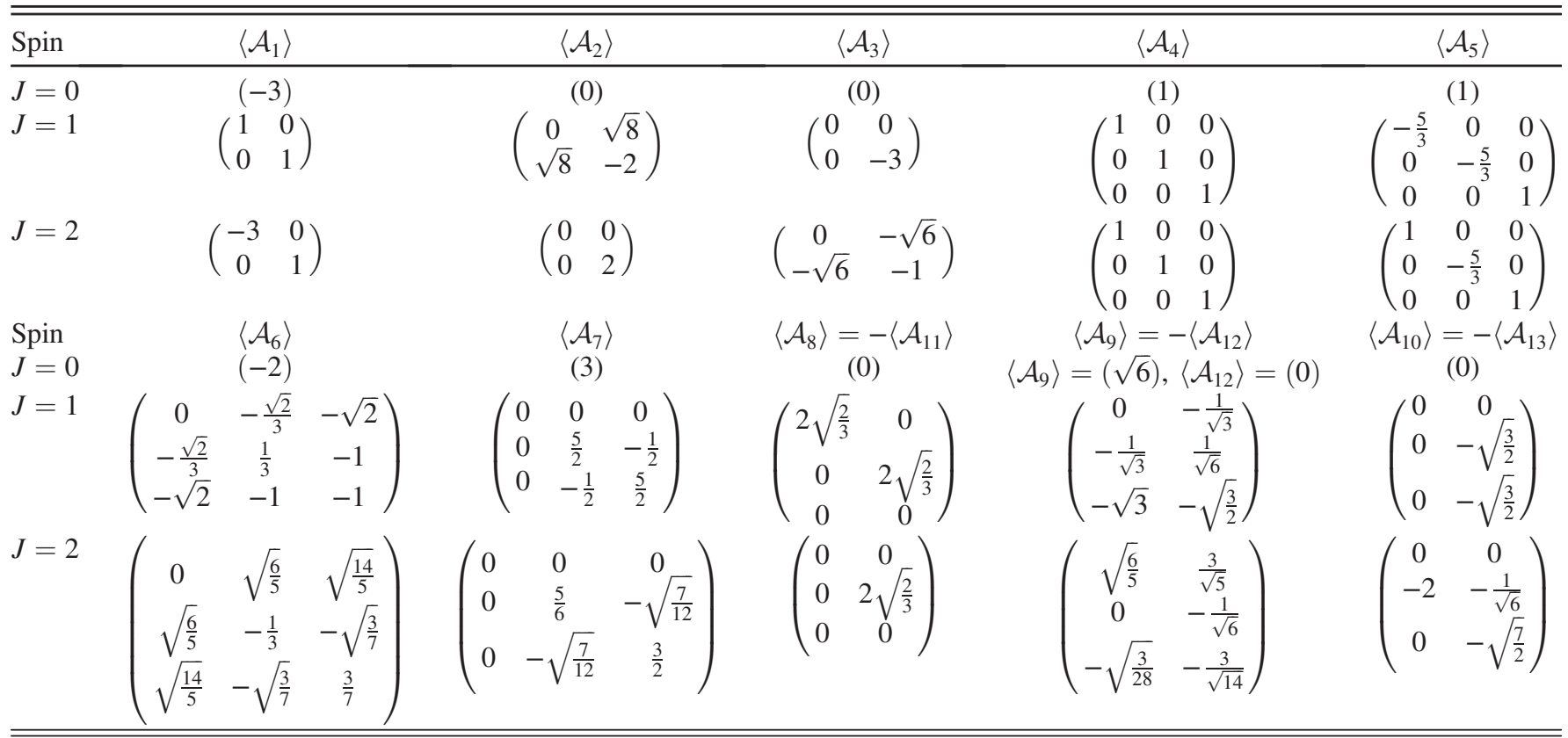

In the above potentials, we also define several spin-spin, spin-orbit, and tensor force operators, including

$$
\begin{gathered}
\mathcal{A}_{1}=\chi_{4}^{\dagger} \chi_{3}^{\dagger}\left(\boldsymbol{\sigma}_{\mathbf{1}} \cdot \boldsymbol{\sigma}_{\mathbf{2}}\right) \chi_{2} \chi_{1}, \\
\mathcal{A}_{2}=\chi_{4}^{\dagger} \chi_{3}^{\dagger} S\left(\hat{r}, \boldsymbol{\sigma}_{\mathbf{1}}, \boldsymbol{\sigma}_{\mathbf{2}}\right) \chi_{2} \chi_{1}, \\
\mathcal{A}_{3}=\chi_{4}^{\dagger} \chi_{3}^{\dagger}\left(\boldsymbol{\sigma}_{\mathbf{2}} \cdot \boldsymbol{L}\right) \chi_{2} \chi_{1}, \\
\mathcal{A}_{4}=\sum_{a, b}^{m, n} C_{1 / 2, a ; 1, b}^{3 / 2, a+b} C_{1 / 2, c ; 1, d}^{3 / 2, c+d} \chi_{4 a}^{\dagger} \chi_{3}^{\dagger} \chi_{2 m} \chi_{1} \boldsymbol{\epsilon}_{\mathbf{2}}^{\boldsymbol{n}} \cdot \boldsymbol{\epsilon}_{\mathbf{4}}^{\boldsymbol{b} \dagger}, \\
\mathcal{A}_{5}=\sum_{a, b}^{m, n} C_{1 / 2, a ; 1, b}^{3 / 2, a+b} C_{1 / 2, c ; 1, d}^{3 / 2, c+d} \chi_{4 a}^{\dagger} \chi_{3}^{\dagger} i \boldsymbol{\sigma}_{\mathbf{1}} \cdot\left(\boldsymbol{\epsilon}_{\mathbf{2}}^{\boldsymbol{n}} \times \boldsymbol{\epsilon}_{\mathbf{4}}^{\boldsymbol{b} \dagger}\right) \chi_{2 m} \chi_{1}, \\
\mathcal{A}_{6}=\sum_{a, b}^{m, n} C_{1 / 2, a ; 1, b}^{3 / 2, a+b} C_{1 / 2, c ; 1 d}^{3 / 2, c+d} \chi_{4 a}^{\dagger} \chi_{3}^{\dagger} S\left(\hat{r}, \boldsymbol{\sigma}_{\mathbf{1}}, i \boldsymbol{\epsilon}_{\mathbf{2}}^{\boldsymbol{n}} \times \boldsymbol{\epsilon}_{\mathbf{4}}^{\boldsymbol{b} \dagger}\right) \chi_{2 m} \chi_{1}, \\
\mathcal{A}_{7}=\sum_{a, b}^{m, n} C_{1 / 2, a ; 1, b}^{3 / 2, a+b} C_{1 / 2, c ; 1, d}^{3 / 2, c+d} \chi_{4 a}^{\dagger} \chi_{3}^{\dagger} i\left(\boldsymbol{\epsilon}_{\mathbf{2}}^{\boldsymbol{n}} \times \boldsymbol{\epsilon}_{\mathbf{4}}^{\boldsymbol{b} \dagger}\right) \cdot \boldsymbol{L} \chi_{2 m} \chi_{1},
\end{gathered}
$$

$$
\begin{gathered}
\mathcal{A}_{8}=\sum_{m, n} C_{1 / 2, m ; 1, n}^{3 / 2, m+n} \chi_{4 m}^{\dagger} \chi_{3}^{\dagger}\left(\boldsymbol{\sigma}_{\mathbf{1}} \cdot \boldsymbol{\epsilon}_{\mathbf{4}}^{\boldsymbol{n}^{\dagger}}\right) \chi_{2} \chi_{1}, \\
\mathcal{A}_{9}=\sum_{m, n} C_{1 / 2, m ; 1, n}^{3 / 2, m+n} \chi_{4 m}^{\dagger} \chi_{3}^{\dagger} S\left(\hat{r}, \boldsymbol{\sigma}_{\mathbf{1}}, \boldsymbol{\epsilon}_{\mathbf{4}}^{\boldsymbol{n}^{\dagger}}\right) \chi_{2} \chi_{1}, \\
\mathcal{A}_{10}=\sum_{m, n} C_{1 / 2, m ; 1, n}^{3 / 2, m+n} \chi_{4 m}^{\dagger} \chi_{3}^{\dagger}\left(\boldsymbol{L} \cdot \boldsymbol{\epsilon}_{\mathbf{4}}^{\boldsymbol{n} \dagger}\right) \chi_{2} \chi_{1}, \\
\mathcal{A}_{11}=\sum_{m, n} C_{1 / 2, m ; 1, n}^{3 / 2, m+n} \chi_{4 m}^{\dagger} \chi_{3}^{\dagger}\left[\boldsymbol{\sigma}_{\mathbf{1}} \cdot\left(i \boldsymbol{\sigma}_{\mathbf{2}} \times \boldsymbol{\epsilon}_{\mathbf{4}}^{\boldsymbol{n} \dagger}\right)\right] \chi_{2} \chi_{1}, \\
\mathcal{A}_{12}=\sum_{m, n} C_{1 / 2, m ; 1, n}^{3 / 2, m+n} \chi_{4 m}^{\dagger} \chi_{3}^{\dagger} S\left(\hat{r}, \boldsymbol{\sigma}_{\mathbf{1}}, i \boldsymbol{\sigma}_{\mathbf{2}} \times \boldsymbol{\epsilon}_{\mathbf{4}}^{\boldsymbol{n} \dagger}\right) \chi_{2} \chi_{1}, \\
\mathcal{A}_{13}=\sum_{m, n} C_{1 / 2, m ; 1, n}^{3 / 2, m+n} \chi_{4 m}^{\dagger} \chi_{3}^{\dagger}\left[\boldsymbol{L} \cdot\left(i \boldsymbol{\sigma}_{\mathbf{2}} \times \boldsymbol{\epsilon}_{\mathbf{4}}^{\boldsymbol{n} \dagger}\right)\right] \chi_{2} \chi_{1},
\end{gathered}
$$

with

$$
S(\hat{r}, \boldsymbol{a}, \boldsymbol{b})=3(\hat{r} \cdot \boldsymbol{a})(\hat{r} \cdot \boldsymbol{b})-\boldsymbol{a} \cdot \boldsymbol{b}
$$

In Table VI, we present the corresponding matrix elements, which are obtained by sandwiching these operators between the relevant spin-orbit wave functions. 
[1] R. Aaij et al. (LHCb Collaboration), Observation of $J / \psi p$ Resonances Consistent with Pentaquark States in $\Lambda_{b}^{0} \rightarrow J / \psi K^{-} p$ Decays, Phys. Rev. Lett. 115, 072001 (2015).

[2] H. X. Chen, W. Chen, X. Liu, and S. L. Zhu, The hiddencharm pentaquark and tetraquark states, Phys. Rep. 639, 1 (2016).

[3] X. Liu, An overview of $X Y Z$ new particles, Chin. Sci. Bull. 59, 3815 (2014).

[4] A. Hosaka, T. Iijima, K. Miyabayashi, Y. Sakai, and S. Yasui, Exotic hadrons with heavy flavors: X, Y, Z, and related states, Prog. Theor. Exp. Phys. 2016, 062C01 (2016).

[5] D. Alde et al. (IHEP-Brussels-Los Alamos-Annecy(LAPP) Collaboration), Evidence for a $1^{-+}$Exotic Meson, Phys. Lett. B 205, 397 (1988).

[6] V. M. Abazov et al. (D0 Collaboration), Evidence for a $B_{s}^{0} \pi^{ \pm}$State, Phys. Rev. Lett. 117, 022003 (2016).

[7] V. M. Abazov et al. (D0 Collaboration), Study of the $X^{ \pm}(5568)$ state with semileptonic decays of the $B_{s}^{0}$ meson, Phys. Rev. D 97, 092004 (2018).

[8] R. Aaij et al. (LHCb Collaboration), Search for Structure in the $B_{s}^{0} \pi^{ \pm}$Invariant Mass Spectrum, Phys. Rev. Lett. 117, 152003 (2016); Publisher's Note 118, 109904(E) (2017).

[9] A. M. Sirunyan et al. (CMS Collaboration), Search for the $\mathrm{X}(5568)$ State Decaying into $\mathrm{B}_{\mathrm{s}}^{0} \pi^{ \pm}$in Proton-Proton Collisions at $\sqrt{s}=8 \mathrm{TeV}$, Phys. Rev. Lett. 120, 202005 (2018).

[10] T. A. Aaltonen et al. (CDF Collaboration), A Search for the Exotic Meson $X(5568)$ with the Collider Detector at Fermilab, Phys. Rev. Lett. 120, 202006 (2018).

[11] R. Aaij et al. (LHCb Collaboration), Observation of the Doubly Charmed Baryon $\Xi_{c c}^{++}$, Phys. Rev. Lett. 119, 112001 (2017).

[12] L. Meng, N. Li, and S. L. Zhu, Deuteron-like states composed of two doubly charmed baryons, Phys. Rev. D 95, 114019 (2017).
[13] L. Meng, N. Li, and S. 1. Zhu, Possible hadronic molecules composed of the doubly charmed baryon and nucleon, arXiv:1707.03598.

[14] R. Chen, A. Hosaka, and X. Liu, Prediction of triple-charm molecular pentaquarks, Phys. Rev. D 96, 114030 (2017).

[15] Y. R. Liu and M. Oka, $\Lambda_{c} N$ bound states revisited, Phys. Rev. D 85, 014015 (2012).

[16] M. Bando, T. Kugo, S. Uehara, K. Yamawaki, and T. Yanagida, Is the $\rho$ Meson a Dynamical Gauge Boson of Hidden Local Symmetry?, Phys. Rev. Lett. 54, 1215 (1985).

[17] R. Machleidt, The high precision, charge dependent Bonn nucleon-nucleon potential (CD-Bonn), Phys. Rev. C 63, 024001 (2001).

[18] R. Machleidt, K. Holinde, and C. Elster, The Bonn meson exchange model for the nucleon nucleon interaction, Phys. Rep. 149, 1 (1987).

[19] X. Cao, B. S. Zou, and H.S. Xu, Phenomenological analysis of the double pion production in nucleon-nucleon collisions up to $2.2 \mathrm{GeV}$, Phys. Rev. C 81, 065201 (2010).

[20] C. Patrignani et al. (Particle Data Group), Rev. Part. Phys., Chin. Phys. C 40, 100001 (2016).

[21] N. A. Tornqvist, From the deuteron to deusons, an analysis of deuteron-like meson meson bound states, Z. Phys. C 61, 525 (1994).

[22] N. A. Tornqvist, On deusons or deuteron-like meson meson bound states, Nuovo Cimento Soc. Ital. Fis. 107A, 2471 (1994).

[23] R. Chen, X. Liu, and A. Hosaka, Heavy molecules and one- $\sigma / \omega$-exchange model, Phys. Rev. D 96, 116012 (2017).

[24] R. Chen, A. Hosaka, and X. Liu, Searching for possible $\Omega_{c}$-like molecular states from meson-baryon interaction, Phys. Rev. D 97, 036016 (2018).

[25] E. Klempt, F. Bradamante, A. Martin, and J. M. Richard, Antinucleon nucleon interaction at low energy: Scattering and protonium, Phys. Rep. 368, 119 (2002).

[26] S. K. Choi et al. (Belle Collaboration), Observation of a Narrow Charmonium-like State in Exclusive $B^{ \pm} \rightarrow$ $K^{ \pm} \pi^{+} \pi^{-} J / \psi$ Decays, Phys. Rev. Lett. 91, 262001 (2003). 